\title{
Macroalgal assemblage structure on a coral reef in Nanwan Bay in southern Taiwan
}

\author{
Chuan-Chuan Tsai ${ }^{1, a}$, Saou-Lien Wong ${ }^{2}$, Jui- \\ Sheng Chang ${ }^{3, b}$, Ray-Lien Hwang', Chang-Feng \\ Dai $^{3}$, Yi-Chuan Yu${ }^{1}$, Yuang-Tay Shyu ${ }^{4}$, Fuu \\ Sheu ${ }^{4}$ and Tse-Min Lee ${ }^{1, *}$ \\ 1 Institute of Marine Biology, National Sun Yat-sen \\ University, Kaohsiung, Taiwan 804, Republic of China \\ email: tmlee@mail.nsysu.edu.tw \\ 2 Department of Aquaculture, National Ping-Tung \\ Polytechnic University, Pingtung, Taiwan 912, Republic \\ of China \\ ${ }^{3}$ Institute of Oceanography, National Taiwan University, \\ Taipei, Taiwan 106, Republic of China \\ ${ }^{4}$ Department of Horticulture, National Taiwan University, \\ Taipei, Taiwan 106, Republic of China \\ ${ }^{*}$ Corresponding author
}

\begin{abstract}
Four-season surveys of macroalgal assemblage structure on a coral reef in southern Taiwan were performed by transect investigation from October 1999 through August 2000. Species richness increased over time, and percentage cover and biomass peaked in April. Non-metric multidimensional scaling ordination analysis of species similarities between stations showed three distinct clusters corresponding to the upper reef flat, the lower reef flat and the reef slope. The macroalgal assemblage on the lower reef flat was further grouped into two clusters corresponding to the warmer months (October 1999 and August 2000) and the cooler months (January and April 2000), suggesting the macroalgal assemblage is structured primarily by zone and secondarily by season. Species richness, percentage cover, biomass, species diversity $\left(H^{\prime}\right)$ and evenness $\left(J^{\prime}\right)$ were highest on the lower reef flat, reflecting greater complexity in the macroalgal assemblage in this zone. However, species diversity and evenness on the lower reef flat and the reef slope did not show seasonal variations. Species diversity in the upper reef flat decreased in the warmer months due to the dominance of an unknown red alga in October 1999 and Enteromorpha linza in August 2000. The results of ANOSIM tests showed that species structure was different not only between zones but also between seasons, and the SIMPER analyses showed that, in the warmer months, Gracilaria coronopifolia, Enteromorpha linza, Laurencia spp. and Hypnea charoides were the species that separated the lower reef flat assemblage from other assemblages. In the same way, the unknown red alga (for

\footnotetext{
a Present address: Kaohsiung Municipal Ming-Yi Junior High School, Kaohsiung, Taiwan 804, Republic of China.

b Present address: Center of General Education, Yu Da University, Miaoli, Taiwan 361, Republic of China.
}

October 1999), Enteromorpha linza (for August 2000), Halimeda spp., Mastrophora rosea and Portieria hornemannii were the species responsible for differences in structure between the upper reef flat and the reef slope. In the cooler months, the species responsible for differences in structures between the upper reef flat and the other two zones were Sargassum spp., Gracilaria coronopifolia, Enteromorpha linza and Padina australis, and those responsible for differences between the upper reef flat and the reef slope were Hypnea charoides, Halimeda spp., Mastophora rosea, Enteromorpha linza and Portieria hornemannii. The present investigation suggests that macroalgal abundance and structure on a coral reef in Nanwan Bay in southern Taiwan have marked spatial zonation and seasonal variations, and Sargassum on the lower reef flat has become the most abundant alga, as compared to 12 years ago.

Keywords: assemblage; macroalgae; seasonality; zonation.

\section{Introduction}

Along with tropical rainforests, coral reefs are the most diverse marine ecosystems with highest productivity on the earth. Macroalgae, one of the components of the coral reef ecosystem, are usually inconspicuous on welldeveloped reefs where nutrient concentrations are low and grazing pressure is high. In the past few years, several lines of evidence have shown that many coral reefs in tropical coastal waters of the western and central Pacific, Indian and western Atlantic Oceans have undergone shifts from coral to macroalgal dominance (Littler et al. 1992, Naim 1993, Hughes 1994, Lapointe 1997). The shift of coral reefs to algal domination causes a dramatic decline in biodiversity in the reef ecosystem (Hughes 1994, Andres and Witman 1995). Thus, understanding the macroalgal abundance is an important aspect of the ecological, environmental, aesthetic and socio-economic value of reefs.

Coastal regions along Hengchuan Peninsula have the best developed coral reef ecosystem in southern Taiwan and face increasing pressure from expanded urban development and tourism. Most nearshore reefs in Hengchuan Peninsula are characterized by a high abundance of fleshy macroalgae and a low abundance of herbivorous fishes (Dai 1997). Changes in macroalgal assemblages are considered to be a consequence of habitat modification mediated by physical and/or anthropogenic disturbances (Banner 1974, Smith et al. 1981, RodriguezPrieto and Polo 1996). For example, increasing watercolumn nutrient concentrations seem to play a role in macroalgal blooming on Reunion Island, Indian Ocean (Cuet et al. 1988) and in the Caribbean and Florida coast- 
A. Map of study site

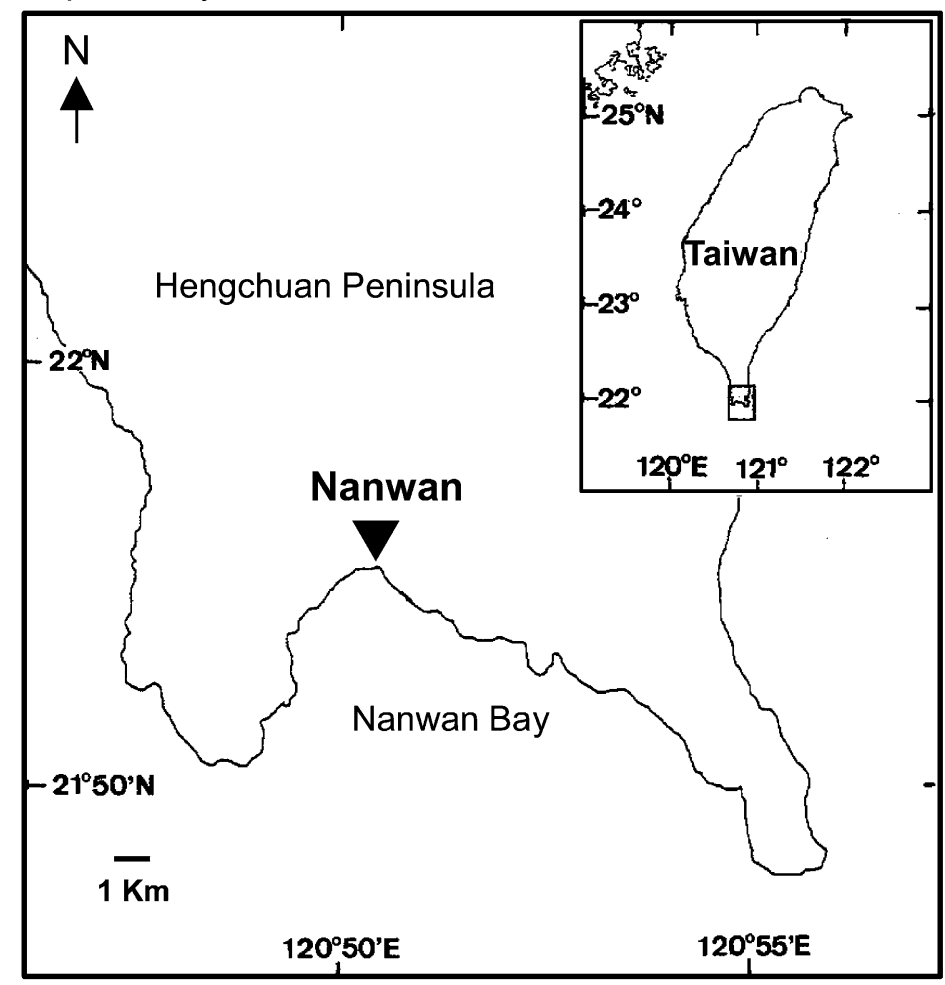

B. Sampling station $(\downarrow)$ on the transect

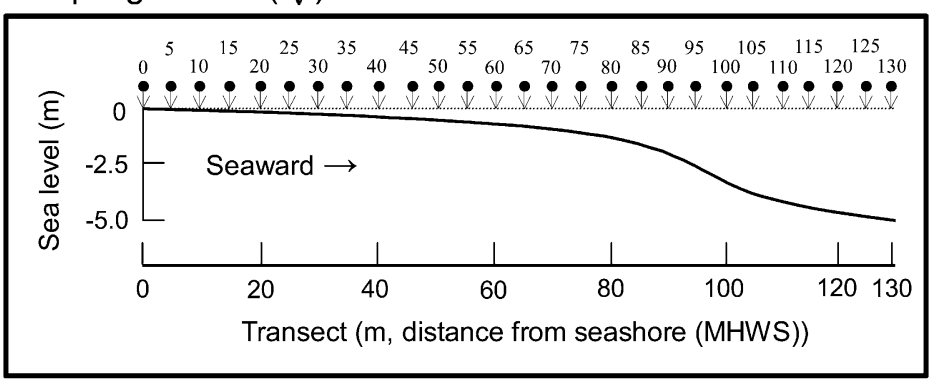

Figure 1 Map of Nanwan Bay in Hengchuan Peninsula in southern Taiwan with (A) location of the coral reef studied and (B) a sketch of sampling stations on each transect perpendicular to the shoreline [in a direction from shoreline (MHWS) to seaward].

al regions (Lapointe and O'Connell 1989, Bell 1992). Apparently, the growth of macroalgae out competes corals after changes driven by natural disturbance or human activities (McCook 1999, Schaffelke 1999, McCook et al. 2001), and highly abundant macroalgae, especially erect fleshy algae, are a sign of reef degradation (Done 1992, Hughes 1994). In addition, because seaweeds tend to integrate the effects of long-term exposure to adverse conditions, macroalgal assemblages are widely used to characterize and monitor benthic communities. Although qualitative observations have shown that the abundance of fleshy macroalgae in southern Taiwan has increased over the past ten years (Dai 1997), quantitative investigations on macroalgal abundance and species compositions have not been done on the reefs of this area. Hence, the present investigation [part of two collaborative coral reef conservation studies supported by the National Science Council (from 1995-1998 and from 1999-2000) and the Council of Agriculture (from 2002-2004), Taiwan, Republic of China] has been undertaken on a coral reef in Nanwan Bay in order to determine the seasonal and spatial variations in macroalgal assemblage compositions in tropical Taiwan waters.

\section{Materials and methods}

\section{Study site and environmental characteristics}

The study site is a nearshore coral reef (GPS: $21^{\circ} 56^{\prime} 00^{\prime \prime} \mathrm{N}$; $120^{\circ} 50^{\prime} 10^{\prime \prime} \mathrm{E}$ ) in Nanwan Bay (Figure 1A), which is located on the western side of Hengchuan Peninsula surrounded by the Pacific Ocean to the east, the Bashi Channel to the south, and the Taiwan Straits to the west. This reef which is located beside the "Nanwan" sand beach on Nanwan Bay has a horizontal width of 1,200 m, comprising an intertidal region, approximately 50-130 m broad, and a subtidal macroalgal region, approximately 35-270 $\mathrm{m}$ broad and a depth gradient of 0-12 $\mathrm{m}$ [below mean high water springs (MHWS)] on a seaward gradient. In the upper intertidal region, there are two large intertidal pools of ca. $20-40 \mathrm{~m}$ width and $30-40 \mathrm{~m}$ length 


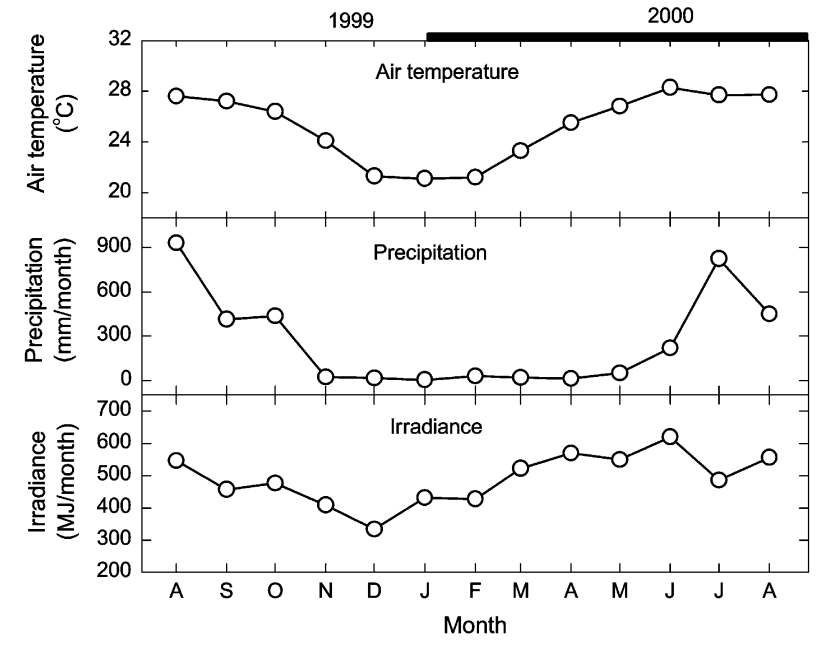

Figure 2 Climate data for Hengchuan Peninsula from August 1999 to August 2000.

covered with fine sediments and seagrass [Thalassia hemprichii (Ehrenberg) Ascherson]. In the subtidal regions, there is a reef flat bordered by a sloping coralline rocky stretch ca. 80-90 $\mathrm{m}$ away from the coast line. Two drainage outlets are significant sewage sources in this reef, especially during the rainy period (from April to July).

The data obtained from the Central Weather Bureau of the Republic of China (1960-1990) show that the mean annual air temperature in Hengchuan Peninsula is $25^{\circ} \mathrm{C}$; the mean monthly air temperature is low $\left(20.3^{\circ} \mathrm{C}\right)$ in January and high $\left(28.3^{\circ} \mathrm{C}\right)$ in July. The annual average relative humidity is $77 \%$, maximum precipitation (mean annual precipitation is $1964.9 \mathrm{~mm}$ ) and typhoons usually occur in May-September. The prevailing northeasterly winds occur during September-March, and during this monsoon period, the hydrological regimen is affected by strong dry winds from land to seaward. During September 1999-August 2000, the mean monthly air temperature was $<25^{\circ} \mathrm{C}$ from November 1999 through March 2000 , monthly cumulative precipitation was low from
November 1999 through June 2000 and irradiance was lower than $500 \mathrm{MJ} / \mathrm{m}^{2}$ from September 1999 through February 2000 (Figure 2).

\section{Estimation of macroalgal cover, biomass and species composition}

To characterize the spatial changes in macroalgal assemblage compositions, four transects (each transect was $130 \mathrm{~m}$ long) perpendicular to the shoreline were set at $100-\mathrm{m}$ intervals and on each transect, sampling stations were marked at 5-m intervals running from the average high tide mark (MHWS) in a seaward direction; the $0 \mathrm{~m}$ station was made to coincide with the average high tide mark and 27 stations were set for each transect line (Figure 1B). At each station, species abundance, in terms of percentage cover, was calculated as the sampling surface covered in vertical projection by the species using a $50 \times 50 \mathrm{~cm}$ quadrat, and the total macroalgal cover was the sum of all species cover. The macroalgal cover in different vegetation layers (erect layer, encrusting layer and turf) was recorded, and all seaweeds in a $50 \times 50 \mathrm{~cm}$ quadrat were scraped free for estimation of macroalgal compositions and biomass. Species identification was determined using a microscope. Macroalgal cover and biomass were determined in October 1999, January 2000, April 2000 and August 2000 for the analysis of temporal changes in macroalgal assemblage structure.

\section{Determination of seawater temperature, salinity, irradiance and nutrient concentrations}

Our preliminary investigations in April and August 1999 indicated that three zones corresponding to different air exposure time can be found from the average high tide mark towards the low tide mark: an upper reef flat (0-20 $\mathrm{m}$ from MHWS) with average daily exposure time $>10 \mathrm{~h}$, a lower reef flat (25-80 m from MHWS) with average daily exposure time $<6 \mathrm{~h}$, and a reef slope (85-130 m from MHWS) without exposure to air. Envi-

Table 1 Changes in water temperature, salinity, irradiance, and DIN and SRP concentrations during surveys.

\begin{tabular}{|c|c|c|c|c|c|c|}
\hline & & $\begin{array}{l}\text { Water temperature } \\
\left({ }^{\circ} \mathrm{C}\right)\end{array}$ & $\begin{array}{l}\text { Salinity } \\
\text { (psu) }\end{array}$ & $\begin{array}{l}\text { Irradiance } \\
\left(\mathrm{MJ} / \mathrm{m}^{2}\right)\end{array}$ & $\begin{array}{l}\text { DIN } \\
(\mu \mathrm{M})\end{array}$ & $\begin{array}{l}\text { SRP } \\
(\mu \mathrm{M})\end{array}$ \\
\hline Oct. 1999 & $\begin{array}{l}\text { Upper reef flat } \\
\text { Lower reef flat } \\
\text { Reef slope }\end{array}$ & $\begin{array}{l}27.08 \pm 0.66 a \\
27.10 \pm 0.68 a \\
26.67 \pm 0.68 a\end{array}$ & $\begin{array}{l}35.88 \pm 0.98 a \\
38.13 \pm 3.60 a \\
36.04 \pm 3.20 a\end{array}$ & $\begin{array}{l}988.17 \pm 125.85 a \\
794.67 \pm 69.70 a b \\
464.17 \pm 53.80 c\end{array}$ & $\begin{array}{c}15.37 \pm 12.69 \mathrm{a} \\
6.24 \pm 7.12 \mathrm{a} \\
8.33 \pm 4.32 \mathrm{a}\end{array}$ & $\begin{array}{l}0.88 \pm 2.16 \\
0.00 \\
0.00\end{array}$ \\
\hline Jan. 2000 & $\begin{array}{l}\text { Upper reef flat } \\
\text { Lower reef flat } \\
\text { Reef slope }\end{array}$ & $\begin{array}{l}24.97 \pm 0.59 a \\
23.83 \pm 0.98 b \\
22.62 \pm 0.46 c\end{array}$ & $\begin{array}{l}36.25 \pm 2.96 a \\
36.67 \pm 2.46 a \\
36.04 \pm 1.66 a\end{array}$ & $\begin{array}{l}957.33 \pm 81.92 a \\
796.67 \pm 108.97 a \\
424.33 \pm 87.20 b\end{array}$ & $\begin{array}{l}2.95 \pm 1.08 a \\
1.75 \pm 0.65 a \\
1.15 \pm 0.39 a\end{array}$ & $\begin{array}{l}0.00 \\
0.00 \\
0.00\end{array}$ \\
\hline Apr. 2000 & $\begin{array}{l}\text { Upper reef flat } \\
\text { Lower reef flat } \\
\text { Reef slope }\end{array}$ & $\begin{array}{l}23.00 \pm 0.32 a \\
22.85 \pm 0.37 a \\
22.32 \pm 0.33 b\end{array}$ & $\begin{array}{l}37.50 \pm 0.00 \mathrm{a} \\
37.71 \pm 0.51 \mathrm{a} \\
37.50 \pm 0.00 \mathrm{a}\end{array}$ & $\begin{array}{l}973.17 \pm 49.66 a \\
807.50 \pm 26.90 b \\
447.33 \pm 67.66 c\end{array}$ & $\begin{array}{l}8.87 \pm 1.94 a \\
8.76 \pm 3.05 a \\
8.67 \pm 3.30 a\end{array}$ & $\begin{array}{l}0.06 \pm 0.07 a \\
0.09 \pm 0.06 a \\
0.21 \pm 0.43 a\end{array}$ \\
\hline Aug. 2000 & $\begin{array}{l}\text { Upper reef flat } \\
\text { Lower reef flat } \\
\text { Reef slope }\end{array}$ & $\begin{array}{l}31.33 \pm 0.75 a \\
29.08 \pm 1.02 b \\
27.17 \pm 0.78 c\end{array}$ & $\begin{array}{l}35.00 \pm 0.00 a \\
33.33 \pm 4.08 a \\
33.33 \pm 4.08 a\end{array}$ & $\begin{array}{l}842.17 \pm 105.82 a \\
461.17 \pm 58.28 b \\
265.83 \pm 46.65 c\end{array}$ & $\begin{array}{l}4.88 \pm 5.11 a \\
0.93 \pm 1.20 a \\
2.56 \pm 2.98 a\end{array}$ & $\begin{array}{l}0.00 \\
0.00 \\
0.00\end{array}$ \\
\hline
\end{tabular}

Data are presented as means $\pm S E(n=6)$ and different letters indicate significant differences at $p<0.05$ (Tukey's test). DIN=dissolved inorganic nitrogen $\left(\mathrm{NO}_{3}{ }^{-}+\mathrm{NO}_{2}{ }^{-}+\mathrm{NH}_{4}{ }^{+}\right)$; $\mathrm{SRP}=$ soluble reactive phosphorus. 
ronmental factors including seawater temperature, salinity, irradiance (above the algal canopy) and seawater nutrient concentrations were determined randomly in 6 sampling stations for each zone (Table 1). The upper reef flat was 0-0.5 m deep (MHWS), the lower reef flat was approximately 1-2 $\mathrm{m}$ deep (MHWS), and the reef slope approximately $2.5-5 \mathrm{~m}$ deep. Collected seawater samples were immediately transported to the laboratory under low temperature and then stored at $-70^{\circ} \mathrm{C}$ until analysis. After filtering through Whatman CF/G papers $(0.45 \mu \mathrm{m})$, seawater $\mathrm{NO}_{2}{ }^{-}$and $\mathrm{NO}_{3}{ }^{-}$concentrations were determined according to Strickland and Parsons (1972) and $\mathrm{NH}_{4}{ }^{+}$concentrations were determined according to Parsons et al. (1984). Dissolved inorganic nitrogen (DIN) concentrations were the sum of $\mathrm{NO}_{3}{ }^{-}+\mathrm{NO}_{2}{ }^{-}+\mathrm{NH}_{4}{ }^{+}$concentrations. Soluble reactive phosphorus (SRP) concentrations were determined according to Murphy and Riley (1962). The detection limits for seawater $\mathrm{NO}_{2}{ }^{-}, \mathrm{NO}_{3}{ }^{-}$, $\mathrm{NH}_{4}{ }^{+}$and SRP concentrations were $0.75,0.5,0.5$ and $0.25 \mathrm{~nm}$ in $0.5 \mathrm{ml}$ seawater, respectively.

\section{Data analysis}

Environmental factors including seawater temperature, salinity, irradiance and nutrient concentrations were tested for difference using two-way analysis of variance (ANOVA, SAS statistical software package $v$ 8.0, SAS Ltd., NC, USA) with season and zone as fixed factors. Cochran's C-test was used to test heterogeneity of variance. The data for seawater temperature, salinity and SRP concentrations were log $(x+1)$-transformed. Tukey's test was used for multiple comparisons among means from significant ANOVA tests (Day and Quinn 1989).

Univariate indices and multivariate analysis were used to compare the macroalgal assemblage compositions between stations and between seasons [using the Plymouth Routines in Multivariate Ecological Research (PRIMER) statistical software package, v. 5 (Clarke and Warwick 1994)]. For each station, the average data of 4 replicates (the data collected on each line transect were treated as replicates) were used for analyses. The similarity matrix of species compositions (\% cover without data transformation) was classified by hierarchical agglomerative clustering using the unweighted pair group mean arithmetic (UPGMA) linkage method and was ordinated using non-metric multidimensional scaling (MDS) analysis. Analysis of macroalgal assemblage structure was carried across the upper reef flat (5-20 m stations), the lower reef flat (30-80 m stations) and the reef slope (95-130 $\mathrm{m}$ stations). To measure attributes of community structure between seasons and between zones, several univariate indices including the number of species, the Shannon-Wiener species diversity index, $\mathrm{H}^{\prime}$ (Shannon and Weaver 1949) (by log e in the calculation) and evenness, Pielou's J' (Pielou 1975) (by log e in the calculation), were calculated and tested for difference using two-way (season, zone) and one-way (zone) analysis of variance (ANOVA, SAS statistical software package v 8.0, SAS Ltd., NC, USA). Cochran's C-test was used to determine homogeneity of variance. Tukey's test was used for multiple comparisons among means from significant ANOVA tests (Day and Quinn 1989). Macroalgal assemblages were compared among stations by means of hierarchical agglomerative cluster analysis and MDS (Kruskal and Wish 1978) of species \% cover using the Bray-Curtis similarity measure (Bray and Curtis 1957). Diversity profiles were also drawn using $k$-dominance curves to extract information on patterns of relative species abundance and dominance (Lambshead et al. 1983). The differences in macroalgal assemblage structure between seasons and between zones was tested using two-way analysis of similarity (ANOSIM) (Clarke and Warwick 1994), and the species mainly responsible for differences between zones in each season were determined by the similarity percentage breakdown procedure (SIMPER) (Clarke 1993).

\section{Results}

\section{Environmental factors}

Environmental factors had zonation and seasonal variations (Table 1). Seawater temperature [log $(x+1)$-transformed] showed seasonal (ANOVA; $F_{3,60}=355.28$, $\mathrm{p}<0.0001)$ and zonation $\left(\mathrm{F}_{2,60}=47.22, \mathrm{p}<0.0001\right)$ variations; seawater temperatures in October 1999 and August 2000 were higher than those in January and April 2000 (Tukey, $p<0.05$ ) while in August 2000, seawater temperature in the upper reef flat was higher than those in both the lower reef flat and the reef slope (Tukey, $p<0.05)$. Salinity $[\log (x+1)$-transformed] showed seasonal variations $\left(F_{3,60}=6.67, p=0.0006\right)$ but did not show zonation variations (ANOVA; $F_{3,60}=0.43, p=0.6538$ ); salinity was low in August 2000 (Tukey, $p<0.05$ ). Irradiance showed seasonal (ANOVA; $F_{3,60}=46.02, p<0.0001$ ) and zonation $\left(F_{2,60}=297.31, p<0.0001\right)$ variations; irradiance was low in August 2000 (Tukey, $\mathrm{p}<0.05$ ) and irradiance in the reef slope was significantly lower than that in the upper reef flat (Tukey, $p<0.05$ ). DIN concentrations showed seasonal variations (ANOVA; $F_{3,60}=22.61$, $\mathrm{p}<0.0001$ ) with higher concentrations in October 1999 and April 2000 (Tukey, $p<0.05$ ). Although DIN concentrations did not show zonation variation (ANOVA; $F_{2,60}=2.96$, $p=0.0595$ ), average DIN concentrations in the upper reef flat were higher than those in other zones. Soluble reactive phosphorus concentrations did not show seasonal (ANOVA; $\left.F_{3,60}=0.59, p=0.5582\right)$ or zonation $\left(F_{2,60}=1.08\right.$, $\mathrm{p}=0.3648$ ) variations. The above data together with the climate data from the Central Weather Bureau of the Republic of China (Figure 2) show that October 1999 and August 2000 were the warmer months and January and April 2000 were the cooler months, and the upper reef flat had higher irradiance and higher temperature, especially in the warmer months.

\section{Macroalgal assemblage structure}

Fifty-eight species were recorded during the surveys (Table 2), 19 Chlorophyta, 10 Phaeophyta, 27 Rhodophyta and 2 Cyanophyta. There were 36 species recorded in October 1999 and January 2000, 45 species in April 2000 and 53 species in August 2000. Erect algae were more abundant than encrusting and turf algae. Enteromorpha linza and Hypnea charoides were the common algae on the upper reef flat, Ulva lactuca, Sargassum spp., Gra- 
Table 2 Macroalgae recorded on a nearshore reef at Nanwan Bay in southern Taiwan during October 1999-August 2000.

\begin{tabular}{|c|c|c|c|c|c|c|}
\hline & & & $\begin{array}{l}\text { Oct. } \\
1999\end{array}$ & $\begin{array}{l}\text { Jan. } \\
2000\end{array}$ & $\begin{array}{l}\text { Apr. } \\
2000\end{array}$ & $\begin{array}{l}\text { Aug. } \\
2000\end{array}$ \\
\hline \multicolumn{7}{|l|}{ Chlorophyta } \\
\hline \multirow[t]{3}{*}{ Ulvales } & Ulvaceae & Enteromorpha linza (Linnaeus) J. Agardh & + & + & + & + \\
\hline & & Ulva lactuca Linnaeus & + & + & + & + \\
\hline & & Ulva reticulata Forsskål & & + & + & + \\
\hline \multirow[t]{4}{*}{ Dasycladales } & Dasycladaceae & Bornetella nitida Sonder & & + & + & + \\
\hline & & Bornetella sphaerica (Zanardini) Solms-Laubach & + & + & & + \\
\hline & & Neomeris annulata Dickie & & + & + & + \\
\hline & & Neomeris van-bosseae M.A. Howe & + & & + & + \\
\hline \multirow[t]{2}{*}{ Cladophorales } & Cladophoraceae & Chaetomorpha linum (O.F. Müller) Kützing & + & & + & + \\
\hline & & Cladophora sp. & + & + & + & + \\
\hline \multirow[t]{5}{*}{ Siphonocladales } & Valoniaceae & Valoniopsis pachynema (G. Martens) Børgesen & + & + & & + \\
\hline & & Dictyosphaeria cavernosa (Forsskål) Børgesen & + & & + & + \\
\hline & Boodleaceae & Boodlea composita (Harvey) F. Brand & + & & + & + \\
\hline & Siphonocladaceae & Boergesenia forbesii (Harvey) Feldmann & & & & + \\
\hline & Anadyomenaceae & Anadyomene wrightii Harvey ex J.E. Gray & + & & + & + \\
\hline \multirow[t]{5}{*}{ Bryopsidales } & Caulerpaceae & Caulerpa brachypus f. parvifolia (Harvey) Cribb & & & & + \\
\hline & Halimedaceae & Halimeda macroloba Decaisne & + & + & + & + \\
\hline & & Halimeda opuntia (Linnaeus) Lamouroux & + & + & + & + \\
\hline & Udoteaceae & Chlorodesmis caespitosa J. Agardh & & & + & + \\
\hline & & Chlorodesmis fastigiata (C. Agardh) Ducker & + & + & + & + \\
\hline \multicolumn{7}{|l|}{ Phaeophyta } \\
\hline \multirow[t]{2}{*}{ Dictyotales } & Dictyotaceae & Dictyota cervicornis Kützing & & + & + & + \\
\hline & & Padina australis Hauck & + & + & + & + \\
\hline \multirow[t]{2}{*}{ Scytosiphonales } & Scytosiphonaceae & Colpomenia sinuosa (Mertens ex Roth) Derbès et Solier & & + & & \\
\hline & & Hydroclathrus clathratus (C. Agardh) Howe & & + & & \\
\hline \multirow[t]{6}{*}{ Fucales } & Sargassaceae & Sargassum berberifolium J. Agardh & + & + & + & + \\
\hline & & Sargassum glaucescens J. Agardh & & + & + & \\
\hline & & Sargassum polycystum C. Agardh & + & + & + & + \\
\hline & & Sargassum sandei Reinbold & & & + & + \\
\hline & & Sargassum siliguosum J. Agardh & + & + & + & + \\
\hline & & Turbinaria ornata (Turner) J. Agardh & + & & & + \\
\hline \multicolumn{7}{|l|}{ Rhodophyta } \\
\hline \multirow[t]{5}{*}{ Nemaliales } & Galaxauraceae & Galaxaura marginata (Ellis et Solander) J.V. Lamouroux & + & + & + & + \\
\hline & & Scinaia pseudojaponica Yamada et Tanaka & & + & + & + \\
\hline & Liagoraceae & Helminthocladia australis Harvey & & + & + & + \\
\hline & & Liagora orientalis J. Agardh & & & + & + \\
\hline & & Yamadaella caenomyce (Decaisne) I.A. Abbott & & + & + & + \\
\hline \multirow[t]{5}{*}{ Gracilariales } & Gracilariaceae & $\begin{array}{l}\text { Gracilaria arcuata Zanardini } \\
\text { Gracilaria chorda Holmes }\end{array}$ & & & + & + \\
\hline & & Gracilaria coronopifolia J. Agardh & + & + & + & + \\
\hline & & Gracilaria sordida W.A. Nelson & + & + & + & + \\
\hline & & Gracilaria sp. & + & + & + & + \\
\hline & & Tricleocarpa fragilis (Linnaeus) Huisman et Townsend & & + & + & \\
\hline Corallinales & Corallinaceae & Jania adhaerens Lamouroux & + & + & + & + \\
\hline & & Mastophora rosea (C. Agardh) Setchell & + & + & + & + \\
\hline Gigartinales & Gigartinaceae & Chondrus ocellatus Holmes & + & & & + \\
\hline & Hypneaceae & Hypnea cervicornis J. Agardh & & & + & + \\
\hline & & Hypnea charoides J.V. Lamoruoux & + & + & + & + \\
\hline & & Hypnea pannosa J. Agardh & + & & + & + \\
\hline & Sarcodiaceae & Sarcodia montagneana (J.D. Hooker et Harvey) J. Agardh & & + & + & + \\
\hline & Solieriaceae & Eucheuma serra (J. Agardh) J. Agardh & & + & + & + \\
\hline & Rhizophyllidaceae & Portieria hornemannii (Lyngbye) P.C. Silva & + & + & + & + \\
\hline Rhodomeniales & Champiaceae & Champia bifida Okamura & & & & + \\
\hline Ceramiales & Rhodomelaceae & Acanthophora spicifera (M. Vahl) Børgesen & + & + & + & + \\
\hline & & Acrocystis nana Zanardini & + & & & + \\
\hline & & Bostrychia tenella (J.V. Lamouroux) J. Agardh & + & & & + \\
\hline & & Laurencia intermedia Yamada & + & + & + & + \\
\hline & & Laurencia papillosa (C. Agardh) Greville & + & + & + & + \\
\hline & & Melanamansia glomerata (C. Agardh) R.E. Norris & & + & + & + \\
\hline & & Vidalia obtusiloba (Merten ex C. Agardh) J. Agardh & + & & + & + \\
\hline Unknown red & & & & & & \\
\hline alga & & & + & + & + & + \\
\hline Cyanophyta & & & & & & \\
\hline Oscillatoriales & Oscillatoriaceae & Symploca hydnoides (Harvey) Kützing & + & & + & + \\
\hline & Stigonemataceae & Brachytrichia quoyi (C. Agardh) Bornet et Flahault & & & & + \\
\hline
\end{tabular}



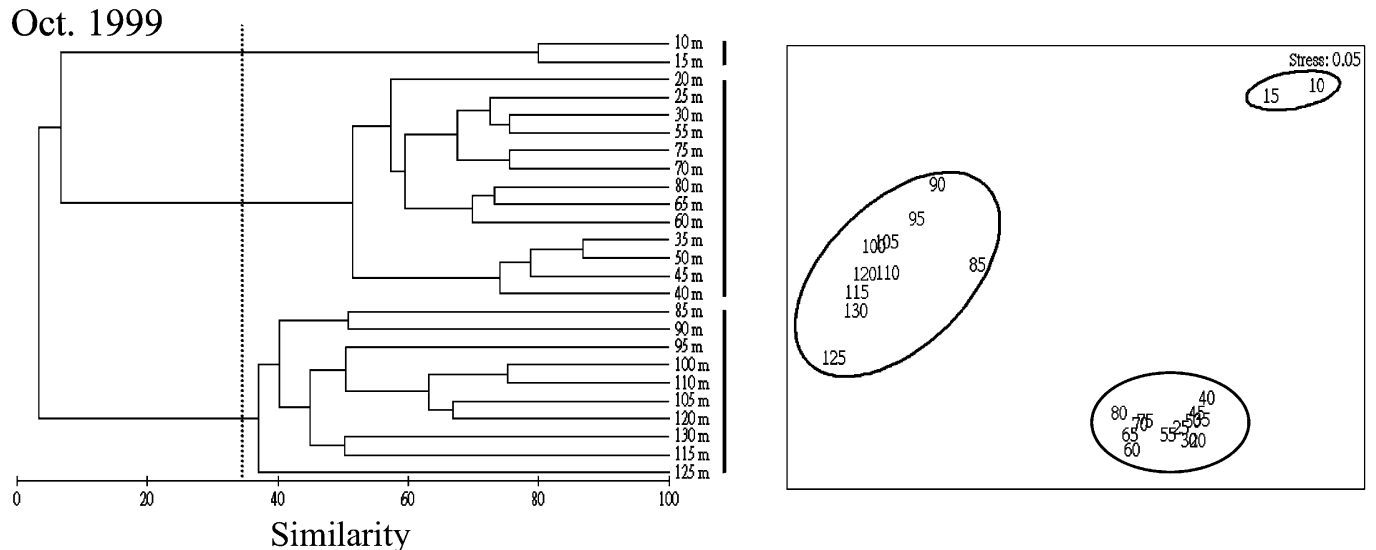

Jan. 2000
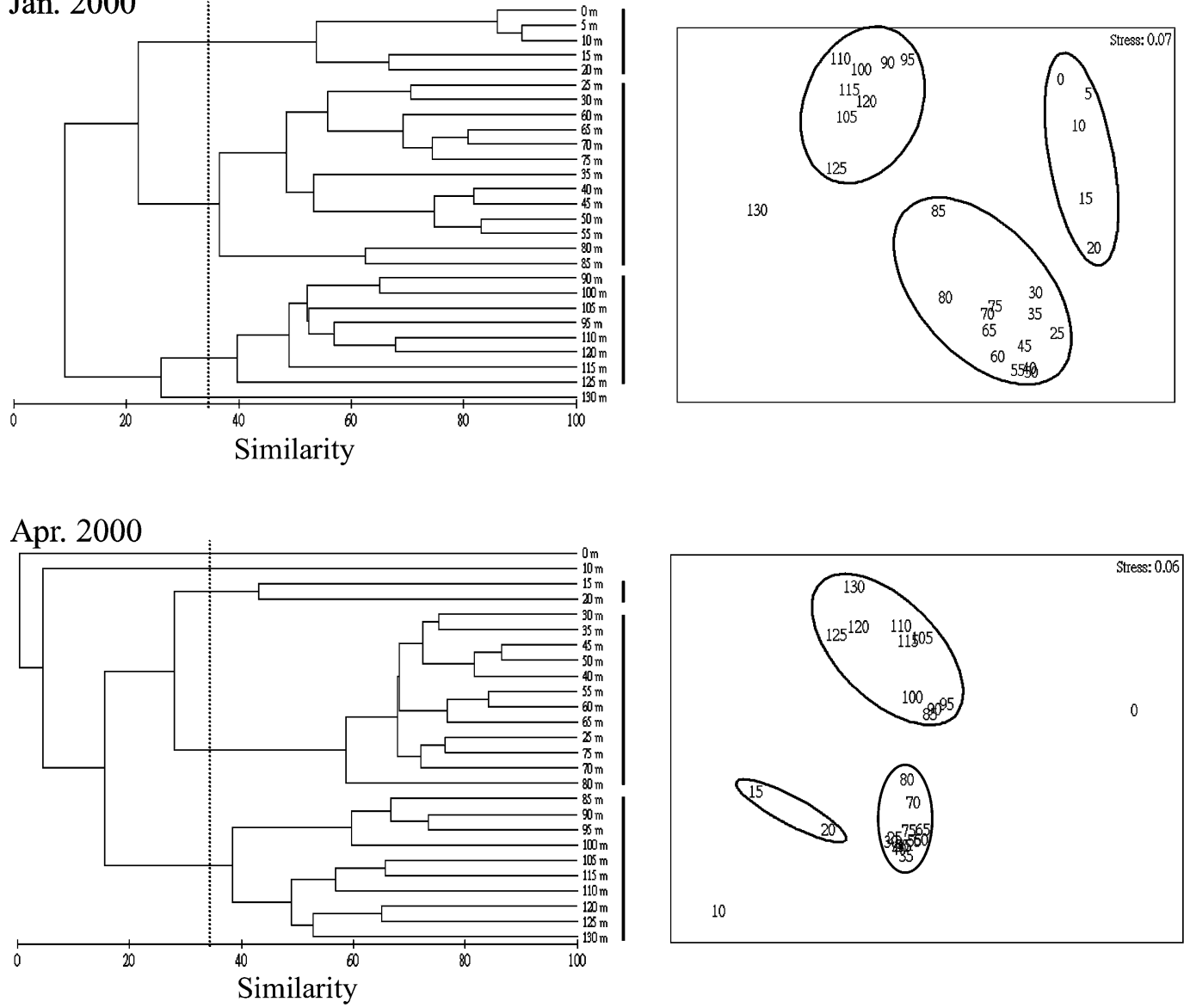

Aug. 2000
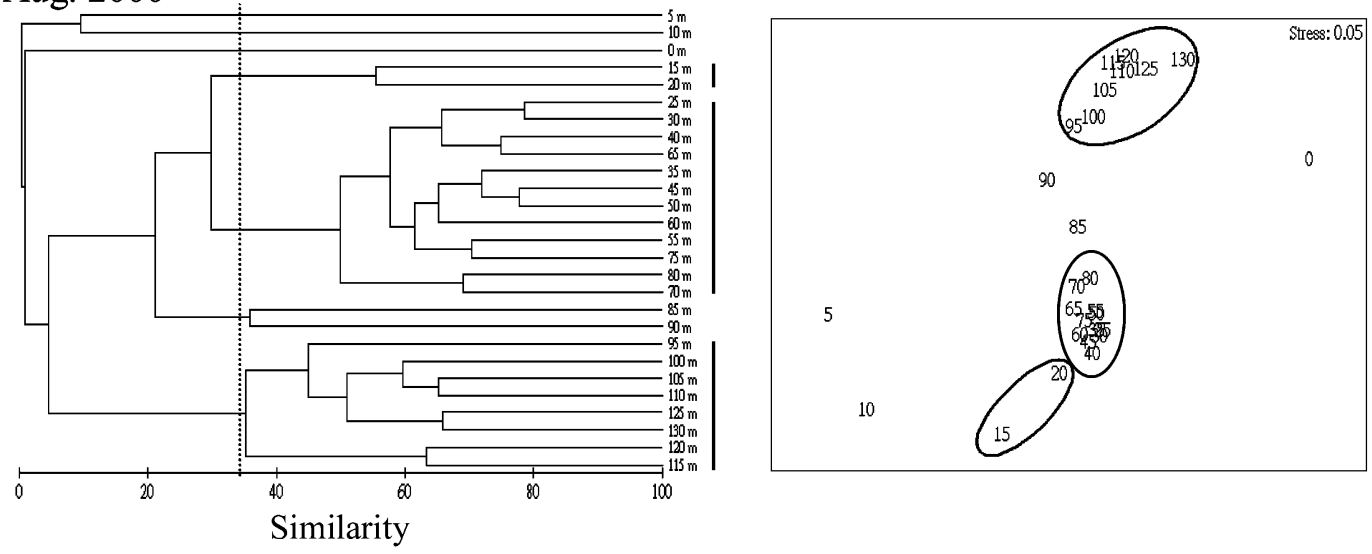

Figure 3 Dendrograms for hierarchical clustering of macroalgal assemblage (similarity threshold at $35 \%$ ) based on Bray Curtis similarities and multidimensional scaling (MDS) ordination.

Number indicates the sampling station, for example, 0 represents the 0-m station. 


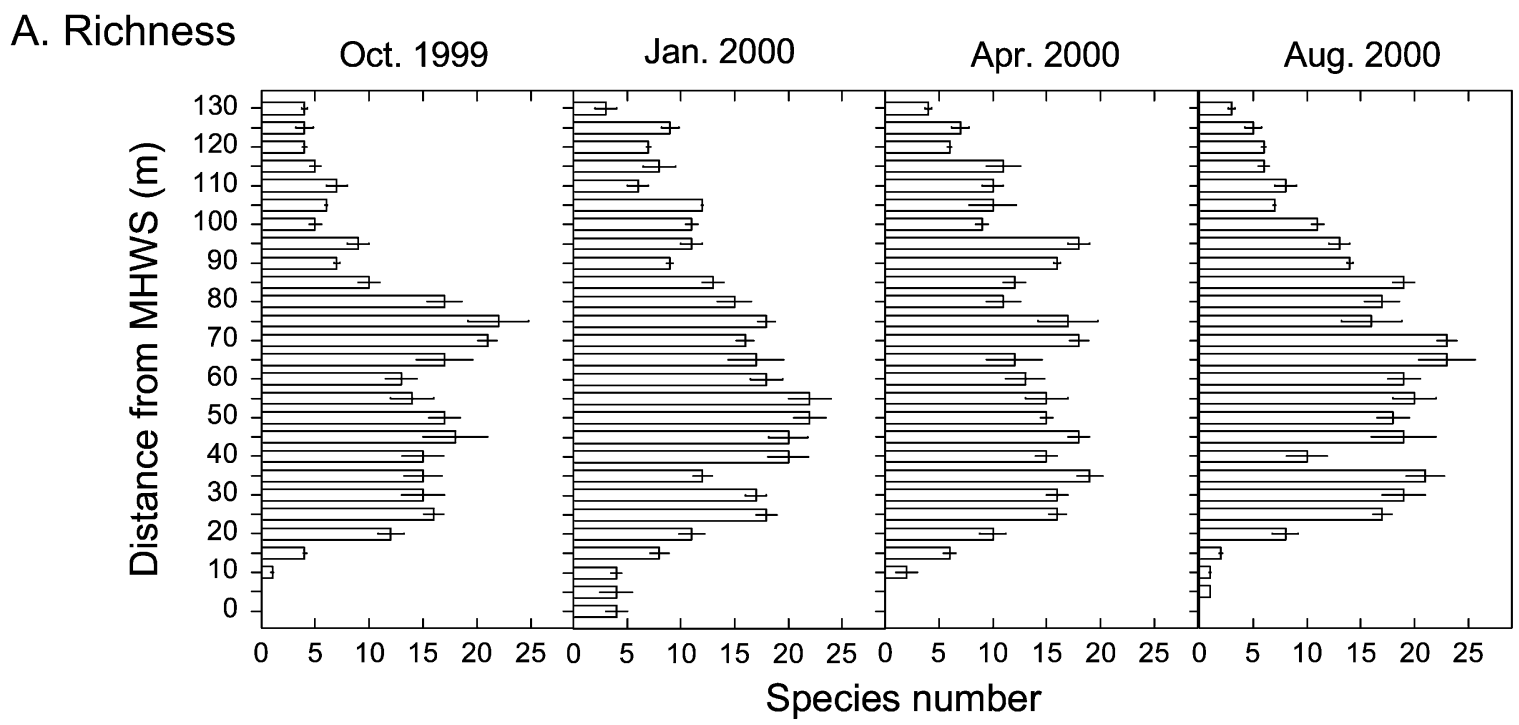

B. Cover

Oct. 1999

Jan. 2000

Apr. 2000

Aug. 2000

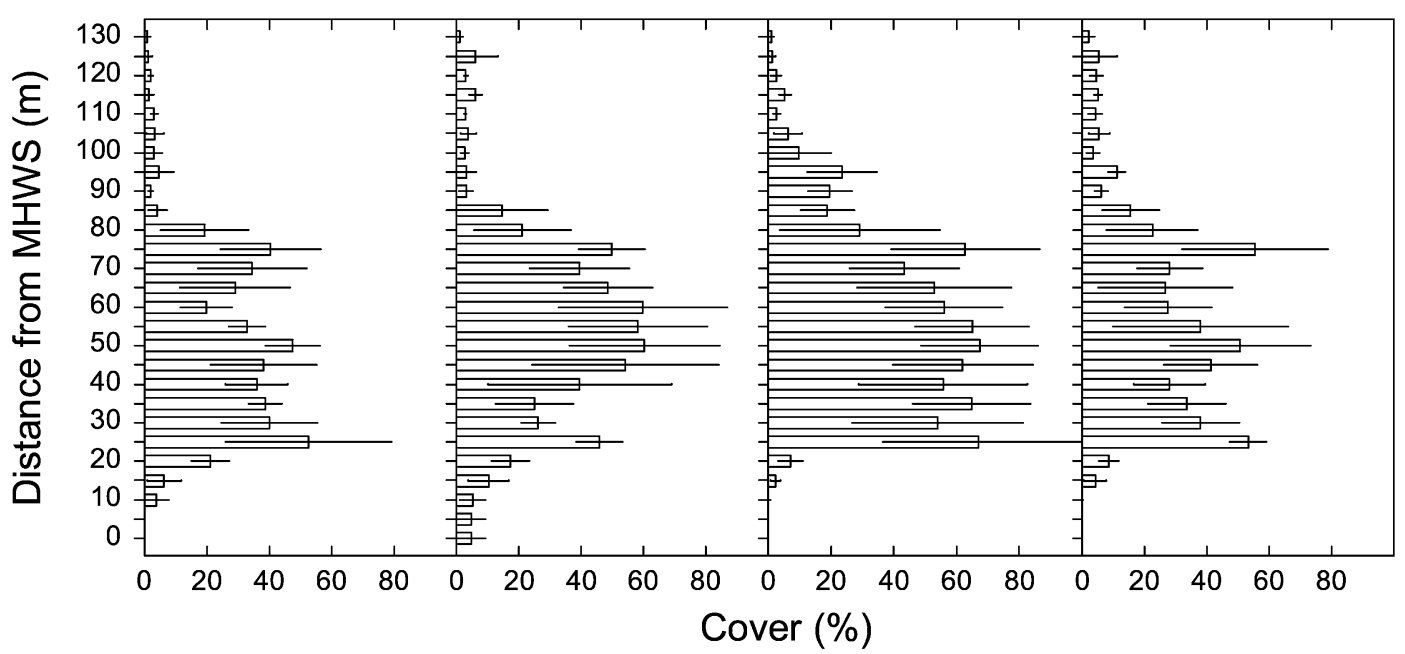

C. Biomass

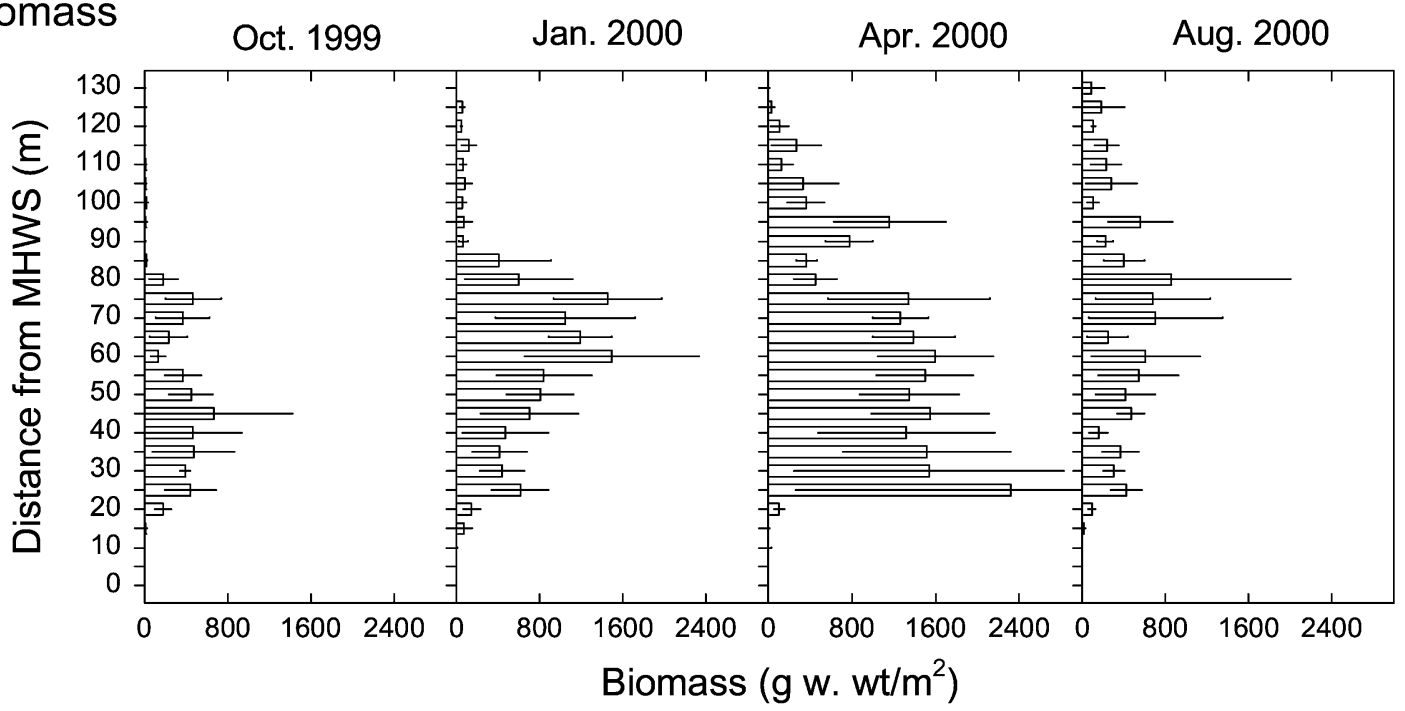

Figure 4 Species richness, percentage cover and biomasses of each station along each transect. Data are presented as means $\pm S D(n=4)$.

cilaria coronopifolia and Laurencia papillosa were abundant on the lower reef flat and Halimeda spp., Portieria hornemannii and Mastophora rosea were abundant on the reef slope. Jania adhaerens, the common turf alga observed in this study coral reef, was abundant on the lower reef flat.

The results from cluster analysis and MDS ordination analysis of species cover (\%) (without data transforma- 
Table 3 Seasonal changes in species richness (species number $\left./ \mathrm{m}^{2}\right)$, cover $(\%)$ and biomass $\left(\mathrm{g} \mathrm{WW} / \mathrm{m}^{2}\right)$ of total macroalgae and abundant macroalgae.

\begin{tabular}{|c|c|c|c|c|c|c|c|}
\hline & & Oct. 1999 & Jan. 2000 & Apr. 2000 & Aug. 2000 & $\mathrm{~F}$ & $\mathrm{P}$ \\
\hline \multirow[t]{3}{*}{ Total macroalgae } & Species richness & $12.77 \pm 6.91 \mathrm{a}$ & $14.73 \pm 5.79 a$ & $13.32 \pm 5.38 a$ & $14.91 \pm 7.78 a$ & 1.05 & 0.6192 \\
\hline & Cover & $31.92 \pm 9.4 b$ & $23.74 \pm 3.38 \mathrm{ab}$ & $36.28 \pm 9.26 a$ & $23.44 \pm 6.87 a b$ & 6.85 & 0.0061 \\
\hline & Biomass & $359.34 \pm 100.95 b$ & $434.27 \pm 50.82 b$ & $948.49 \pm 160.84 a$ & $341.31 \pm 98.82 b$ & 3.67 & 0.0438 \\
\hline \multirow{2}{*}{ Enteromorpha linza } & Cover & $2.47 \pm 4.17 \mathrm{ab}$ & $1.78 \pm 2.16 \mathrm{~b}$ & $1.44 \pm 2.75 b$ & $4.13 \pm 5.02 a$ & 4.07 & 0.0072 \\
\hline & Biomass & $64.08 \pm 4.43 a$ & $43.95 \pm 2.20 a$ & $37.46 \pm 2.94 a$ & $107.85 \pm 5.21 \mathrm{a}$ & 2.15 & 0.0933 \\
\hline \multirow[t]{2}{*}{ Ulva lactuca } & Cover & $1.24 \pm 1.54 \mathrm{ab}$ & $0.87 \pm 1.09 b$ & $0.45 \pm 0.96 b$ & $2.11 \pm 2.89 a$ & 5.30 & 0.0014 \\
\hline & Biomass & $30.35 \pm 1.59 a$ & $19.44 \pm 1.08 a$ & $12.26 \pm 1.03 a$ & $54.24 \pm 3.04 a$ & 0.19 & 0.9040 \\
\hline \multirow[t]{2}{*}{ Halimeda macroloba } & Cover & $0.39 \pm 0.49 a$ & $0.99 \pm 0.76 a$ & $0.63 \pm 1.64 a$ & $0.34 \pm 0.74 a$ & 0.83 & 0.7530 \\
\hline & Biomass & $9.83 \pm 0.51 b$ & $20.76 \pm 0.75 a$ & $8.72 \pm 1.32 b$ & $8.09 \pm 0.79 b$ & 17.05 & 0.0008 \\
\hline \multirow[t]{2}{*}{ Halimeda opuntia } & Cover & $0.31 \pm 0.40 a$ & $0.22 \pm 0.48 a$ & $0.61 \pm 1.04 a$ & $0.66 \pm 1.08 a$ & 0.82 & 0.6807 \\
\hline & Biomass & $6.66 \pm 0.39 c$ & $4.81 \pm 0.49 c$ & $13.16 \pm 1.07 b$ & $16.80 \pm 1.15 a$ & 15.30 & 0.0092 \\
\hline \multirow{2}{*}{ Padina australis } & Cover & $0.03 \pm 0.07 a$ & $0.17 \pm 0.37 a$ & $1.89 \pm 3.05 a$ & $0.03 \pm 0.11 a$ & 0.18 & 0.6279 \\
\hline & Biomass & $0.22 \pm 0.71 \mathrm{ab}$ & $3.23 \pm 7.41 \mathrm{ab}$ & $31.02 \pm 51.79 a$ & $1.34 \pm 3.87 a b$ & 0.99 & 0.0491 \\
\hline \multirow[t]{2}{*}{ Sargassum berberiforium } & Cover & $0.99 \pm 1.40 \mathrm{~b}$ & $3.76 \pm 7.39 b$ & $11.47 \pm 12.54 a$ & $0.06 \pm 0.14 b$ & 17.49 & 0.0001 \\
\hline & Biomass & $25.86 \pm 1.47 b$ & $101.63 \pm 7.84 b$ & $294.88 \pm 13.07 a$ & $1.61 \pm 0.15 b$ & 19.80 & 0.0001 \\
\hline \multirow[t]{2}{*}{ Sargassum polycystum } & Cover & $0.03 \pm 0.07 \mathrm{~b}$ & $5.10 \pm 7.65 a$ & $3.74 \pm 4.82 a$ & $0.07 \pm 0.25 b$ & 16.37 & 0.0001 \\
\hline & Biomass & $0.56 \pm 0.07 c$ & $128.66 \pm 8.04 a$ & $100.33 \pm 4.98 b$ & $2.00 \pm 0.27 c$ & 14.47 & 0.0001 \\
\hline \multirow[t]{2}{*}{ Sargassum siliguosum } & Cover & $0.02 \pm 0.10 \mathrm{~b}$ & $0.05 \pm 0.24 b$ & $3.40 \pm 4.35 a$ & $0.36 \pm 0.67 \mathrm{~b}$ & 16.43 & 0.0001 \\
\hline & Biomass & $0.50 \pm 0.10 \mathrm{~b}$ & $1.38 \pm 0.26 b$ & $91.00 \pm 4.49 a$ & $9.57 \pm 0.71 b$ & 10.73 & 0.0001 \\
\hline \multirow{2}{*}{ Gracilaria coronopifolia } & Cover & $3.72 \pm 4.91 \mathrm{a}$ & $2.28 \pm 2.74 a b$ & $0.91 \pm 1.16 b$ & $2.08 \pm 2.75 b$ & 7.32 & 0.0001 \\
\hline & Biomass & $97.83 \pm 5.12 a$ & $61.43 \pm 2.79 a$ & $23.63 \pm 1.21 b$ & $55.02 \pm 2.87 a$ & 5.51 & 0.0010 \\
\hline \multirow[t]{2}{*}{ Mastophora rosea } & Cover & $0.23 \pm 0.37 a$ & $0.23 \pm 0.42 a$ & $0.22 \pm 0.41 a$ & $0.37 \pm 0.51 a$ & 0.69 & 0.8152 \\
\hline & Biomass & $4.94 \pm 0.37 b$ & $5.94 \pm 0.45 b$ & $5.23 \pm 0.43 b$ & $9.90 \pm 0.53 a$ & 2.92 & 0.0496 \\
\hline \multirow[t]{2}{*}{ Laurencia intermedia } & Cover & $2.98 \pm 3.81 a$ & $0.03 \pm 0.11 a b$ & $0.34 \pm 0.56 a b$ & $1.37 \pm 2.21 \mathrm{a}$ & 1.05 & 0.0435 \\
\hline & Biomass & $75.55 \pm 3.95 a$ & $0.89 \pm 0.11 d$ & $8.80 \pm 0.59 c$ & $35.12 \pm 2.34 b$ & 19.32 & 0.0001 \\
\hline \multirow[t]{2}{*}{ Laurencia papillosa } & Cover & $1.83 \pm 2.29 a$ & $1.17 \pm 1.72 \mathrm{ab}$ & $0.46 \pm 0.55 b$ & $2.30 \pm 3.65 a$ & 4.66 & 0.0032 \\
\hline & Biomass & $47.53 \pm 2.39 a$ & $30.86 \pm 1.81 b$ & $11.81 \pm 0.57 c$ & $56.06 \pm 3.89 a$ & 10.78 & 0.0001 \\
\hline \multirow{2}{*}{ Hypnea charoides } & Cover & $1.67 \pm 2.46 \mathrm{ab}$ & $2.47 \pm 2.33 \mathrm{ab}$ & $1.40 \pm 1.89 b$ & $3.24 \pm 4.11 a$ & 2.86 & 0.0365 \\
\hline & Biomass & $41.37 \pm 2.61 b$ & $60.69 \pm 2.45 b$ & $36.41 \pm 1.98 a b$ & $81.85 \pm 4.37 a$ & 6.12 & 0.0004 \\
\hline \multirow[t]{2}{*}{ Portieria hornemannii } & Cover & $0.13 \pm 0.29 b$ & $0.17 \pm 0.36 \mathrm{~b}$ & $0.63 \pm 0.96 a$ & $0.53 \pm 1.04 a b$ & 2.02 & 0.0465 \\
\hline & Biomass & $1.90 \pm 0.17 c$ & $3.41 \pm 0.35 c$ & $11.53 \pm 0.81 a$ & $9.12 \pm 0.87 \mathrm{~b}$ & 8.71 & 0.0098 \\
\hline
\end{tabular}

Data are presented as means $\pm S D(n=108)$. Different letters indicate significant differences at $p<0.05$ (Tukey).

tion) using Bray Curtis similarity measures discerned three groups corresponding to three zones similar to our preliminary investigations in April and August 1999: an upper reef flat (5-20 $\mathrm{m}$ from MHWS), a lower reef flat (30-80 $\mathrm{m}$ from MHWS) and a reef slope (95-130 $\mathrm{m}$ from MHWS) (Figure 3). The $0 \mathrm{~m}, 25 \mathrm{~m}, 85 \mathrm{~m}$ and $90 \mathrm{~m}$ stations were not included to avoid marginal effects. The data on total macroalgal biomass and percentage cover and species richness (i.e., species number per $\mathrm{m}^{2}$ ) also showed three zones similar to the above patterns (Figure 4).

The data for 108 sampling stations were pooled and averaged for analysis to give an overall picture of seasonal changes in macroalgal abundance: species richness did not show seasonal variations (ANOVA, $F_{3}=1.05$, $p=0.6192$ ) but total macroalgal cover (ANOVA, $F_{3}=6.85$, $p=0.0061$ ) and biomass (ANOVA, $F_{3}=3.67, p=0.0001$ ) were temporally variable, with a peak in April 2000 (Tukey, $\mathrm{p}<0.05$ ) (Table 3). Percentage cover and/or biomasses of abundant macroalgae also showed seasonal variations (Table 3).

Apart from significant seasonal variations, species richness (ANOVA, $\mathrm{F}_{2,78}=161.82, \mathrm{p}<0.0001$ ), percentage cover (ANOVA, $\mathrm{F}_{2,78}=215.18, \mathrm{p}<0.0001$ ) and biomass (ANOVA, $\mathrm{F}_{2,78}=261.33, \mathrm{p}<0.0001$ ) also show zone variations (Figure 5). Species richness, percentage cover and biomass were highest in the lower reef flat. The percentage cover and biomass of common species also showed zoned variation. Enteromorpha linza, Gracilaria coronopifolia, Hypnea charoides and an unknown red alga were common in the upper reef flat, while Ulva lactuca, Sargassum spp. (especially in the stations of $45-70 \mathrm{~m}$ ) and Laurencia spp. were abundant in the lower reef flat. We found that Halimeda macroloba bloomed in the upper reef flat in January 2000. The most abundant alga, Sargassum, bloomed in the cooler months and we have identified 5 species in this survey: S. berberifolium, $S$. polycystum and $S$. siliguosum were abundant and $S$. glaucescens and $S$. sandei were less abundant. The calcified algae Halimeda spp. and Mastophora rosea were abundant on the reef slope.

Evenness (J') showed zonation variations (ANOVA, $\left.F_{2,78}=23.34, p<0.0001\right)$ but did not show seasonal variations (ANOVA, $F_{3,78}=2.28, p=0.1112$ ) (Figure 5). Species diversity $\left(H^{\prime}\right)$ showed zonation (ANOVA, $F_{2,78}=102.81$, $p<0.0001$ ) and seasonal (ANOVA, $F_{3,78}=4.43, p=0.0063$ ) variations (Figure 5). Evenness and species diversity in the upper reef flat showed seasonal variations while those in the lower reef flat and reef slope did not show seasonal variations. In the upper reef flat, evenness and diversity in October 1999 and August 2000 were lower than those in January and April 2000 (Figure 5). The $k$-dominance curves also showed that species diversity was higher in both the lower reef flat and the reef slope than in the upper reef flat, reflecting higher species number on the lower reef flat and the reef slope (Figure 6). 

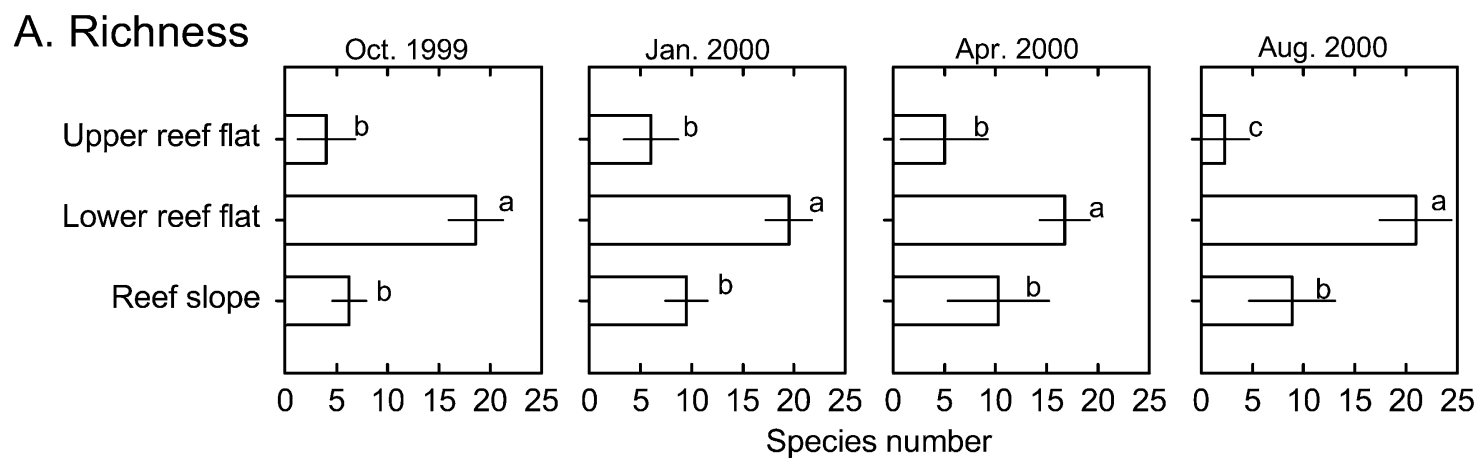

B. Cover
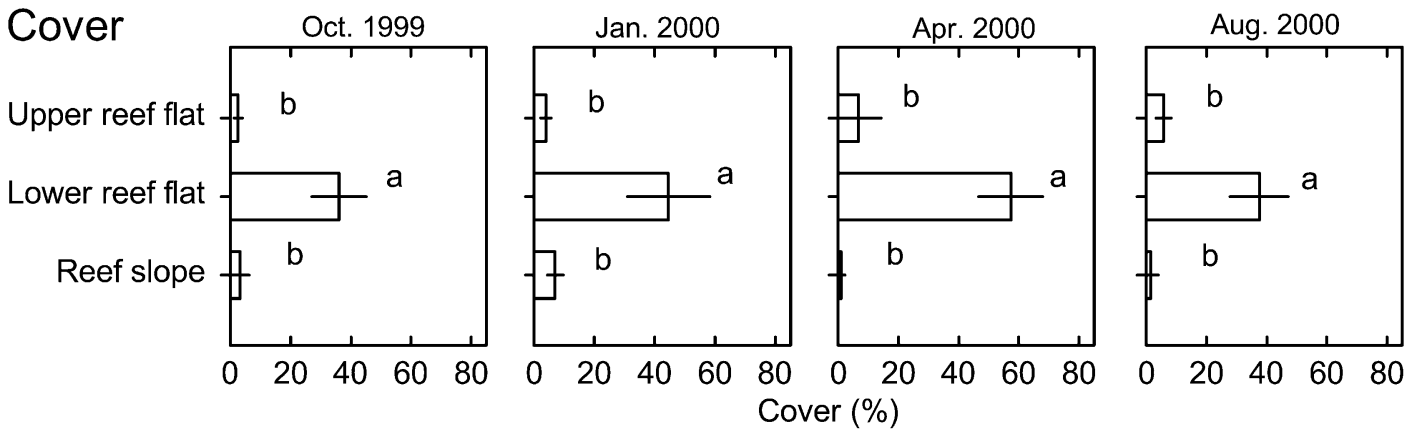

C. Biomass
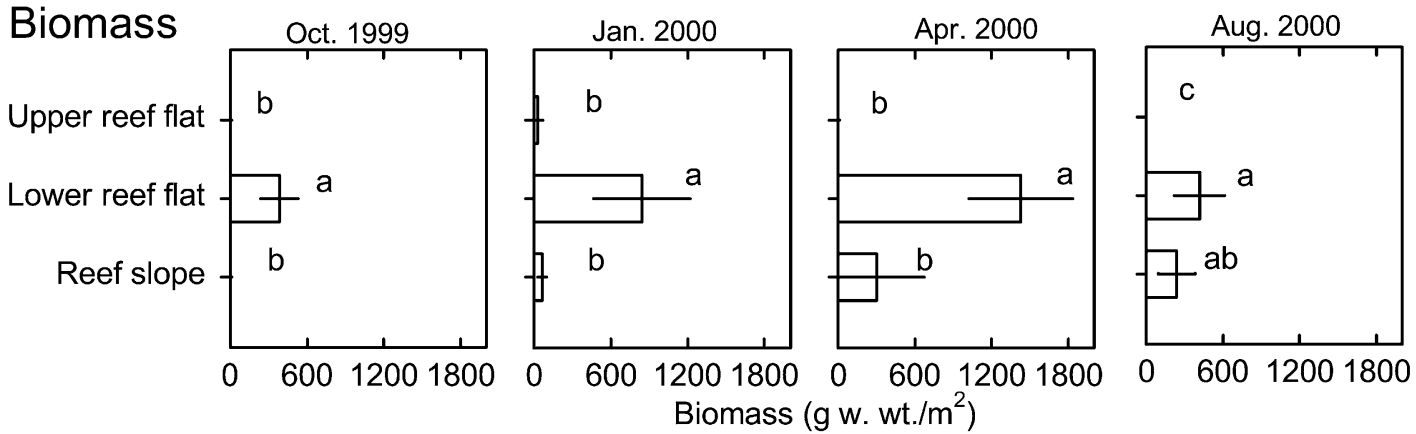

D. Evenness Biomass (g w. wt. $/ \mathrm{m}^{2}$ )
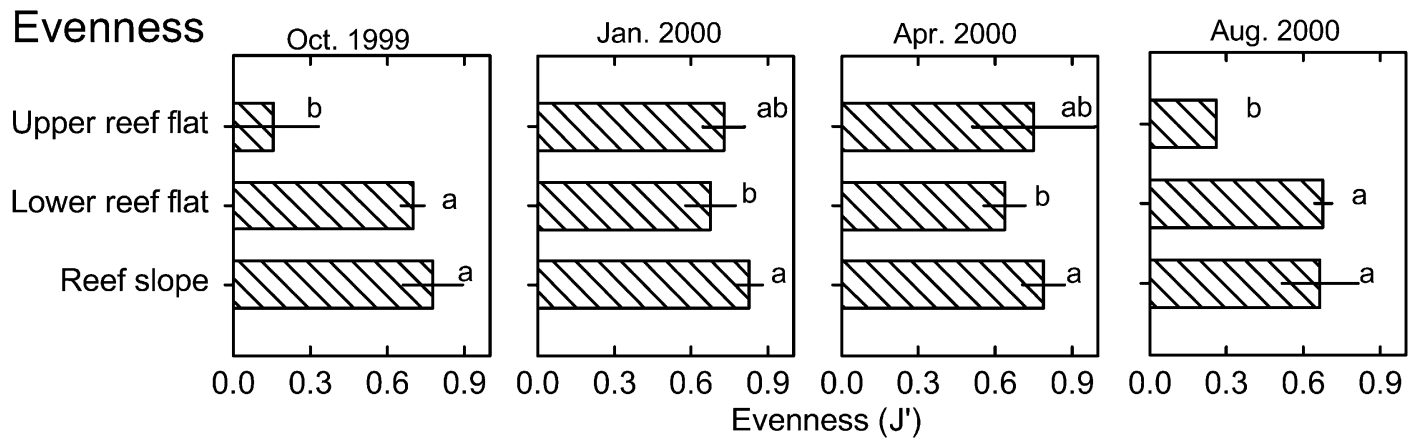

E. Diversity
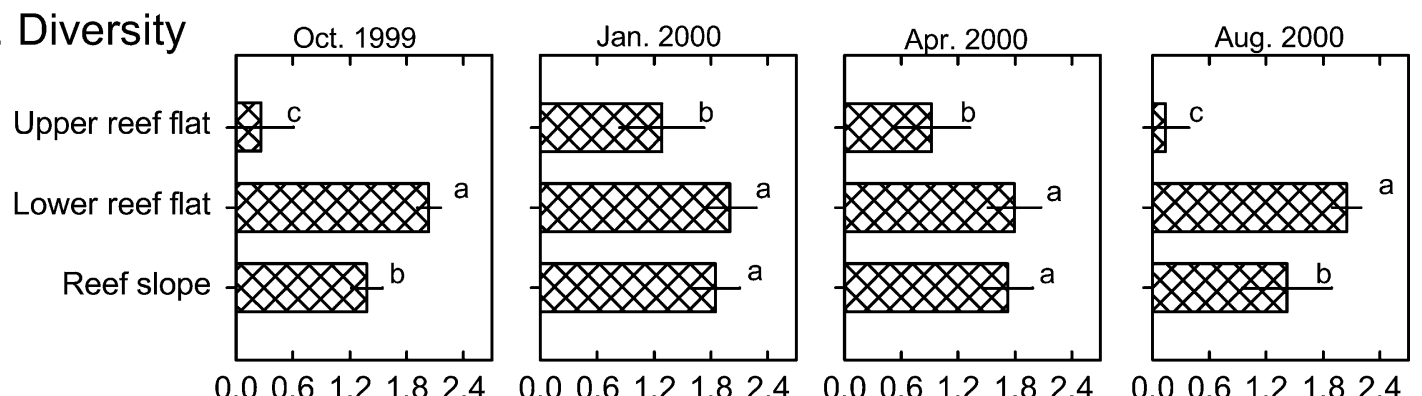

Diversity $\left(\mathrm{H}^{\prime}\right)$

Figure 5 Species richness, percentage cover, biomass, evenness ( $\left.\mathrm{J}^{\prime}\right)$ and species diversity $\left(\mathrm{H}^{\prime}\right)$ on the upper reef flat, the lower reef flat and the reef slope.

Means are indicated and different symbols indicate significant differences at $p<0.05$ (Tukey). 
Oct. 1999

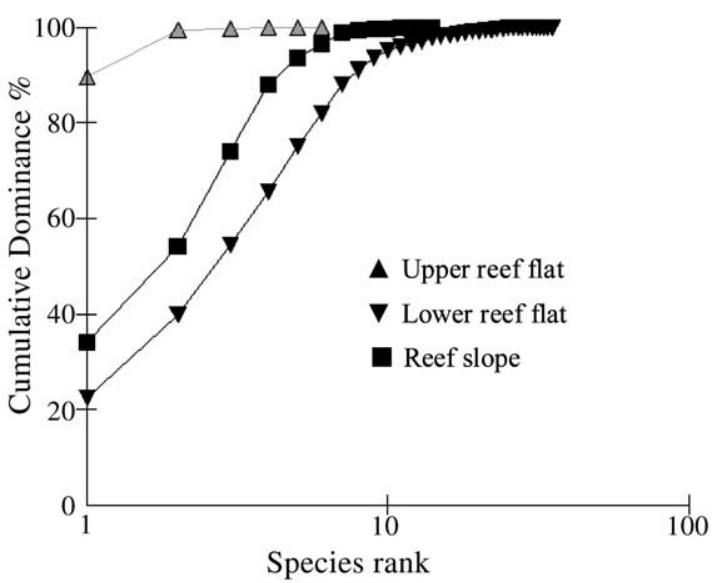

Apr. 2000

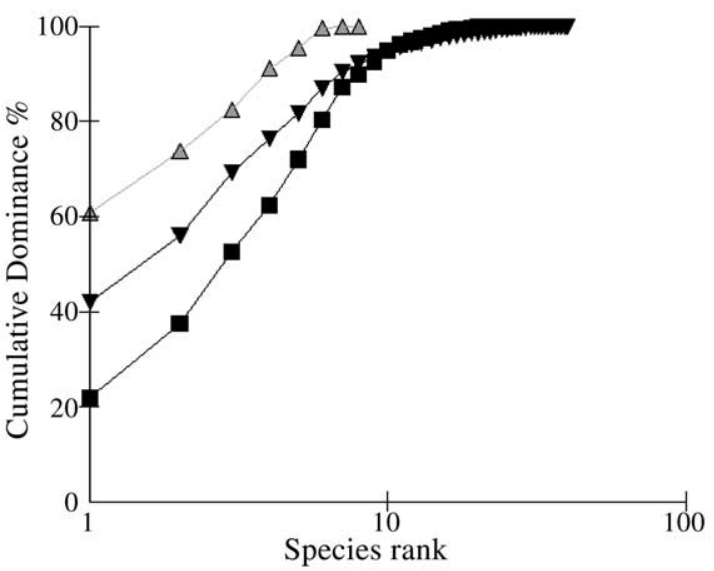

Jan. 2000

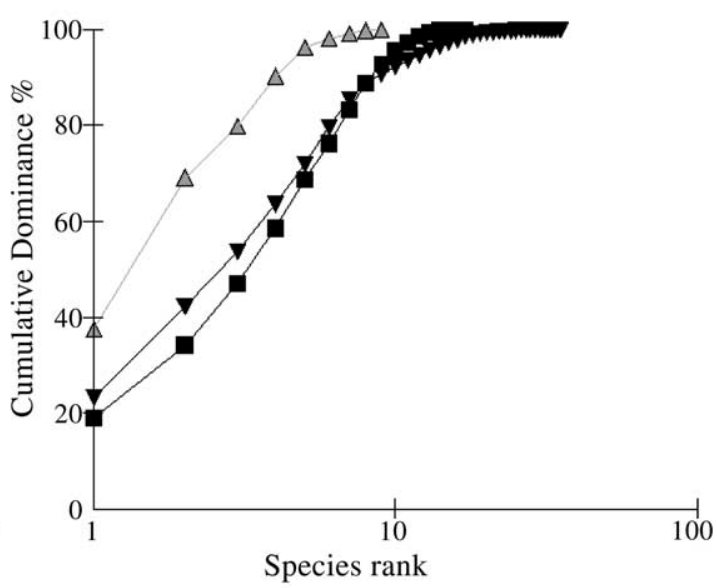

Aug. 2000

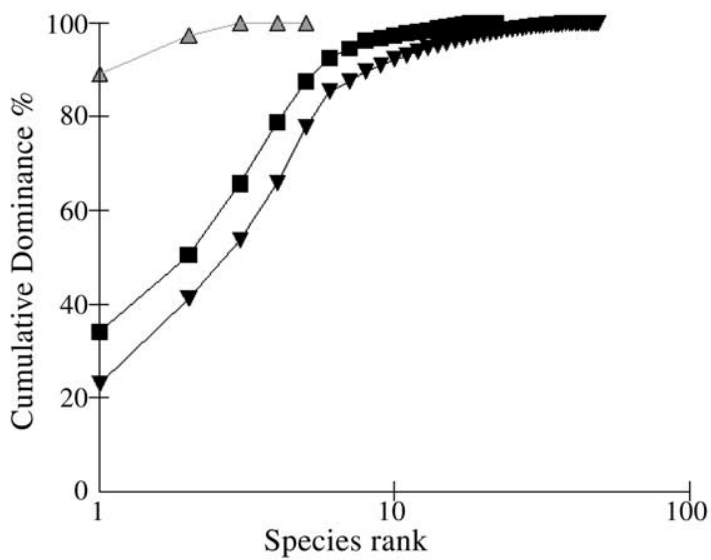

Figure 6 k-dominance curves for macroalgal species abundance in three zones and four seasons.

On the upper reef flat, species diversity in October 1999 and August 2000 was lower than in January and April 2000; this was due to the dominance of the unknown red alga in October 1999 and Enteromorpha linza in August 2000 (Figure 6). It is evident that the macroalgal assemblage is more structured in the lower reef flat compared to the upper reef flat.

The MDS analysis of three zones (the upper reef flat, the lower reef flat and the reef slope) showed that the

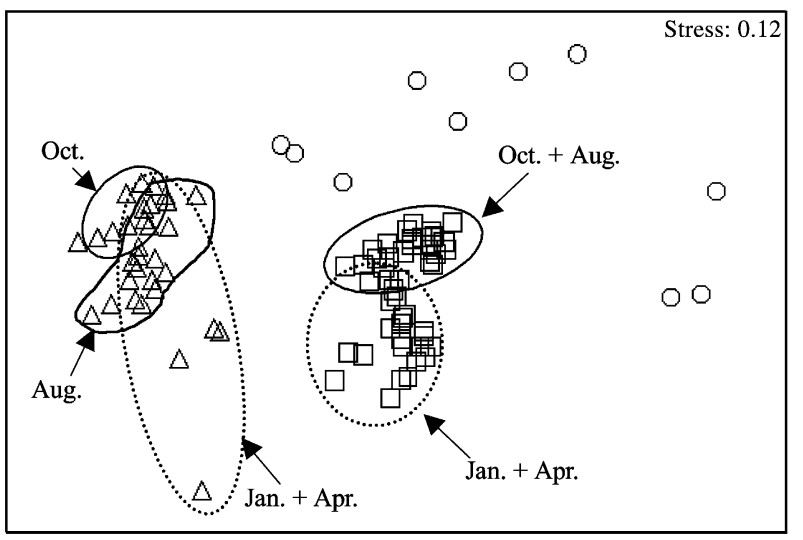

Figure 7 Multidimensional scaling (MDS) ordination of samples taken on the upper reef flat $(\bigcirc)$, the lower reef flat $(\square)$ and the reef slope $(\triangle)$. macroalgal assemblage in the lower reef flat can be grouped into 2 clusters corresponding to the warmer months (October 1999 and August 2000) and the cooler months (January 2000 and April 2000) (Figure 7). It is clear that the macroalgal assemblage is primarily structured by zone and secondarily by season.

The results of ANOSIM tests showed that the species structure was significantly different not only between zones but also between seasons $(p<0.05)$ (Table 4). The Global $R$ value for structure difference between October 1999 and August 2000 was lower than for other comparisons, indicating that the difference between October 1999 and August 2000 was relatively small as compared to other seasons (Table 4).

The results of SIMPER analyses showed that in the warmer months (October 1999 and August 2000), Gracilaria coronopifolia, Enteromorpha linza, Laurencia spp. and Hypnea charoides were the most important taxa separating the lower reef flat assemblage and assemblages in two other zones, and Enteromorpha linza (for August 2000), Halimeda spp., Mastrophora rosea, Portieria hornemannii and the unknown red alga (for October 1999) were the species mostly responsible for differences in assemblage structure between the upper reef flat and the reef slope (Table 5). The data from Table 5 and the percentage cover (Table 6) and biomass (Table 7) showed that in October 1999 and August 2000, Gracilaria coro- 
Table 4 Global R values of two-way (sampling time and zone) crossed ANOSIM tests.

\begin{tabular}{llll}
\hline Global R value for structure difference & & & \\
\hline Between sampling times & zones & & $0.979^{\star}$ \\
\cline { 2 - 4 } Oct. 1999-Jan. 2000 & $0.676^{\star}$ & Upper reef flat-lower reef flat & $0.978^{\star}$ \\
Oct. 1999-Apr. 2000 & $0.798^{\star}$ & Upper reef flat-reef slope & $0.994^{*}$ \\
Oct. 1999-Aug. 2000 & $0.412^{\star}$ & Lower reef flat-reef slope & \\
Jan. 2000-Apr. 2000 & $0.611^{\star}$ & & \\
Jan. 2000-Aug. 2000 & $0.704^{\star}$ & & \\
Apr. 2000-Aug. 2000 & $0.723^{\star}$ & & \\
\hline
\end{tabular}

*Significance level $<5 \%$.

nopifolia, Enteromorpha linza, Laurencia spp. and Hypnea charoides were abundant in the lower reef flat (Tukey, $\mathrm{p}<0.05)$, and Halimeda spp., Mastrophora rosea and Portiera hornemannii were abundant in the reef slope (Tukey, $\mathrm{p}<0.05$ ).

In the cooler months (January and April 2000), the results of SIMPER analyses indicate that the species responsible for difference in structures between the upper reef flat and two zones were Sargassum spp., Gracilaria coronopifolia, Enteromorpha linza and Padina australis, and those responsible for difference between the upper reef flat and the reef slope were Hypnea charoides, Halimeda spp., Mastophora rosea, Enteromorpha linza and Portieria hornemannii (Table 5). Tables 6 and 7 show that in the cooler months, Sargassum spp., Gracilaria coronopifolia and Padina australis had higher percentage cover and biomass in the lower reef flat, while Halimeda spp., Mastophora rosea and Portieria hornemannii had higher percentage cover and biomass in the reef slope (Tukey, $\mathrm{p}<0.05$ ).

\section{Discussion}

The present study shows that macroalgae are highly abundant on a tropical nearshore coral reef in Nanwan Bay in southern Taiwan. Macroalgal biomass appeared to follow a distinct seasonal pattern with blooms in spring (April) and a decline in the warmer months. As compared to a previous study (carried out by random sampling in the lower reef flat at the same site) showing that macroalgal cover and biomass in April of 1987 ranged from $30-70 \%$ and $20-160 \mathrm{~g}$ wet weight $(\mathrm{WW}) / \mathrm{m}^{2}$, respectively (Chiang and Wang 1988), the present results obtained from transect surveys indicate a significant increase in macroalgal biomass over the past 12 years. In April of 2000, macroalgal cover in the lower reef flat ranged from $28.79-88.51 \%$ and biomass ranged from $184.60-770.82 \mathrm{~g} \mathrm{WW} / \mathrm{m}^{2}$. Further, the 4-season survey (during 1987-1988) by Wang and Chiang (1988) reported that the most dominant alga at the lower reef flat in this coral reef was a rhodophyte Hydroclathrus clathratus (32.16\% coverage in April) and there was one Sargassum species (Sargassum polycystum) recorded with a percentage cover of $18.38 \%$ in April of 1987 . However, their survey in 1994 at the same site showed that Sargassum had become the most dominant species in Hengchuan Peninsula (Wang and Chiang 1994). The present study (1999-2000) also found that Sargassum populations had large standing stocks on this coral reef in Nanwan Bay, with maximum percentage cover and biomass of $18.61 \%$ and $486.21 \mathrm{~g} \mathrm{WW} / \mathrm{m}^{2}(64.53 \%$ and $63.68 \%$ of total macroalgal cover and biomass, respectively) in April 2000. So, the spread of Sargassum is evident on a coral reef of Nanwan Bay over the past few years.

The patterns of macroalgal assemblage in Nanwan Bay appeared markedly different across water depths and seasons. Erect algae were the dominant component on this coral reef, and their composition and abundance varied significantly between zones and seasons. The lower reef flat assemblage exhibited higher species number, evenness and diversity as compared to the upper reef flat and the reef slope. Most of the abundant species such as Sargassum spp., Gracilaria coronopifolia, Laurencia spp. and Hypnea charoides occurred largely on the lower reef flat. The Sargassum population in the lower reef flat showed a marked seasonal fluctuation with the maximum abundance in April, with only holdfasts and/or plants with small shoots remaining in the hot summer months.

It is known that the growth and distribution of macroalgae are influenced by physical factors such as temperature, light, salinity and water movements (Lüning 1993, Andrew and Viejo 1998), and increased nutrient concentrations in water columns should also be considered. Our recent investigation provides several lines of evidence showing that increased nutrient input is one of the factors leading to the overgrowth by Sargassum in Nanwan Bay (Hwang et al. 2004). The impact of seawater temperature on Sargassum seasonality has also been shown in Hawaii (DeWreede 1976), the British Isles (Jephson and Gray 1977) and Northern Philippines (Hurtado and Ragaza 1999). Using canonical correlation analysis $(p<0.05)$, temperature was extracted to correlate with primary cover of Sargassum from reefs at Balibago, Luzon, Philippines (Ang 1986). It is also suggested in our recent study that Sargassum seasonality in Nanwan Bay is linked to temporal variations in seawater temperature (Hwang et al. 2004). Possibly, temperature and nutrients interact to affect Sargassum growth in southern Taiwan.

The upper reef flat had lower species number, evenness and diversity; in this zone, Enteromorpha linza was the most common taxon in all seasons, possibly due to its high tolerance to long air exposure times and higher temperature in the upper regions of the intertidal zone. This agrees with the view that Enteromorpha is one of the most stress resistant macroalgae (Littler and Littler 1980). Further, the data on seasonal changes in evenness and species diversity values and $k$-dominance curves in the upper reef flat reveal that the upper reef flat assemblage in the warmer months was less diverse than that in the cooler months, and this is mainly due to the dis- 
Table 5 Results of SIMPER test on percentage contributions of species for significant ANOSIM contrasts.

\begin{tabular}{|c|c|c|c|c|}
\hline Species & Mean abundance & Mean abundance & Contribution (\%) & Cumulative contribution (\%) \\
\hline & \multicolumn{4}{|l|}{ Oct. 1999} \\
\hline & Upper reef flat & Lower reef flat & & \\
\hline Gracilaria coronopifolia & 0.00 & 8.15 & 20.52 & 20.52 \\
\hline Laurencia intermedia & 0.00 & 6.29 & 16.36 & 36.88 \\
\hline Enteromorpha linza & 0.49 & 5.26 & 12.19 & 49.08 \\
\hline \multirow[t]{2}{*}{ Laurencia papillosa } & 0.00 & 3.96 & 10.78 & 59.86 \\
\hline & Upper reef flat & Reef slope & & \\
\hline Unknown red alga & 4.50 & 0.00 & 60.43 & 60.43 \\
\hline Halimeda macroloba & 0.00 & 0.90 & 11.27 & 71.69 \\
\hline Mastophora rosea & 0.00 & 0.53 & 6.88 & 78.58 \\
\hline \multirow[t]{2}{*}{ Halimeda opuntia } & 0.00 & 0.52 & 6.54 & 85.12 \\
\hline & Lower reef flat & Reef slope & & \\
\hline Gracilaria coronopifolia & 8.15 & 0.00 & 21.14 & 21.14 \\
\hline Laurencia intermedia & 6.29 & 0.00 & 16.88 & 38.02 \\
\hline Enteromorpha linza & 5.26 & 0.00 & 12.83 & 50.85 \\
\hline \multirow[t]{3}{*}{ Laurencia papillosa } & 3.96 & 0.00 & 11.16 & 62.01 \\
\hline & \multicolumn{4}{|l|}{ Jan. 2000} \\
\hline & Upper reef flat & Lower reef flat & & \\
\hline Sargassum polycystum & 0.00 & 10.42 & 25.61 & 25.61 \\
\hline Sargassum berberifolium & 0.00 & 8.47 & 18.96 & 44.57 \\
\hline Gracilaria coronopifolia & 0.08 & 5.09 & 12.64 & 57.22 \\
\hline \multirow[t]{2}{*}{ Enteromorpha linza } & 0.73 & 3.48 & 7.89 & 65.10 \\
\hline & Upper reef flat & Reef slope & & \\
\hline Hypnea charoides & 2.63 & 0.00 & 29.90 & 29.90 \\
\hline Halimeda macroloba & 2.21 & 0.49 & 19.65 & 49.55 \\
\hline Mastophora rosea & 0.00 & 0.74 & 7.82 & 57.37 \\
\hline \multirow[t]{2}{*}{ Enteromorpha linza } & 0.73 & 0.00 & 6.47 & 63.84 \\
\hline & Lower reef flat & Reef slope & & \\
\hline Sargassum polycystum & 10.42 & 0.45 & 22.54 & 22.54 \\
\hline Sargassum berberifolium & 8.47 & 0.00 & 17.21 & 39.76 \\
\hline Gracilaria coronopifolia & 5.09 & 0.02 & 11.73 & 51.48 \\
\hline \multirow[t]{3}{*}{ Hypnea charoides } & 4.40 & 0.00 & 9.72 & 61.20 \\
\hline & \multicolumn{4}{|l|}{ Apr. 2000} \\
\hline & Upper reef flat & Lower reef flat & & \\
\hline Sargassum berberifolium & 0.00 & 24.11 & 42.95 & 42.95 \\
\hline Sargassum polycystum & 0.00 & 8.05 & 14.01 & 56.96 \\
\hline Sargassum siliguosum & 0.00 & 7.56 & 13.17 & 70.13 \\
\hline \multirow[t]{2}{*}{ Padina australis } & 0.00 & 4.14 & 6.79 & 76.92 \\
\hline & Upper reef flat & Reef slope & & \\
\hline Halimeda opuntia & 0.00 & 1.50 & 20.13 & 20.13 \\
\hline Enteromorpha linza & 0.88 & 0.00 & 14.91 & 35.04 \\
\hline Portieria hornemannii & 0.00 & 1.08 & 11.70 & 46.74 \\
\hline \multirow[t]{2}{*}{ Mastophora rosea } & 0.00 & 0.65 & 10.22 & 56.96 \\
\hline & Lower reef flat & Reef slope & & \\
\hline Sargassum berberifolium & 24.11 & 0.69 & 39.69 & 39.69 \\
\hline Sargassum polycystum & 8.05 & 0.47 & 12.66 & 52.35 \\
\hline Sargassum siliguosum & 7.56 & 0.03 & 12.46 & 64.81 \\
\hline \multirow[t]{3}{*}{ Padina australis } & 4.14 & 0.00 & 6.48 & 71.29 \\
\hline & \multicolumn{4}{|l|}{ Aug. 2000} \\
\hline & Upper reef flat & Lower reef flat & & \\
\hline Enteromorpha linza & 1.38 & 8.64 & 21.40 & 21.40 \\
\hline Hypnea charoides & 0.13 & 6.79 & 18.26 & 39.67 \\
\hline Gracilaria coronopifolia & 0.00 & 4.58 & 12.54 & 52.21 \\
\hline \multirow[t]{2}{*}{ Laurencia papillosa } & 0.00 & 4.67 & 12.47 & 64.68 \\
\hline & Upper reef flat & Reef slope & & \\
\hline Enteromorpha linza & 1.38 & 0.00 & 14.42 & 14.42 \\
\hline Halimeda macroloba & 0.00 & 0.95 & 13.65 & 28.07 \\
\hline Mastophora rosea & 0.00 & 0.76 & 11.35 & 39.42 \\
\hline Portieria hornemannii & 0.00 & 0.88 & 9.81 & 49.23 \\
\hline & Lower reef flat & Reef slope & & \\
\hline Enteromorpha linza & 8.64 & 0.00 & 20.95 & 20.95 \\
\hline Hypnea charoides & 6.79 & 0.00 & 15.97 & 36.92 \\
\hline Gracilaria coronopifolia & 4.58 & 0.00 & 10.77 & 47.70 \\
\hline Laurencia papillosa & 4.67 & 0.00 & 10.75 & 58.44 \\
\hline
\end{tabular}


Table 6 Cover (\%) (means $\pm S D, n=6$ ) of macroalgal species from SIMPER test.

\begin{tabular}{|c|c|c|c|}
\hline & Upper reef flat & Lower reef flat & Reef slope \\
\hline & Oct. 1999 & & \\
\hline Enteromorpha linza & $2.78 \pm 4.82$ & $5.26 \pm 5.02$ & 0.00 \\
\hline Halimeda macroloba & 0.00 & $0.22 \pm 0.24$ & $0.90 \pm 0.56$ \\
\hline Halimeda opuntia & 0.00 & $0.21 \pm 0.36$ & $0.52 \pm 0.41$ \\
\hline Gracilaria coronopifolia & 0.00 & $8.15 \pm 4.22$ & 0.00 \\
\hline Mastophora rosea & 0.00 & $0.06 \pm 0.10$ & $0.53 \pm 0.49$ \\
\hline Laurencia intermedia & 0.00 & $6.29 \pm 3.26$ & $0.00 \pm 0.01$ \\
\hline Laurencia papillosa & 0.00 & $3.96 \pm 1.80$ & $0.00 \pm 0.01$ \\
\hline \multirow[t]{2}{*}{ Unkown red alga } & $2.29 \pm 2.19$ & $1.11 \pm 1.80$ & 0.00 \\
\hline & Jan. 2000 & & \\
\hline Enteromorpha linza & $9.24 \pm 16.00$ & $3.48 \pm 1.93$ & 0.00 \\
\hline Halimeda macroloba & 0.00 & $0.85 \pm 0.64$ & $0.49 \pm 0.42$ \\
\hline Sargassum berberifolium & 0.00 & $8.47 \pm 9.25$ & $0.00 \pm 0.00$ \\
\hline Sargassum polycystum & 0.00 & $10.42 \pm 8.70$ & $0.45 \pm 0.77$ \\
\hline Gracilaria coronopifolia & $0.18 \pm 0.31$ & $5.09 \pm 1.50$ & $0.02 \pm 0.04$ \\
\hline Hypnea charoides & $4.20 \pm 5.89$ & $4.40 \pm 1.89$ & 0.00 \\
\hline \multirow[t]{2}{*}{ Mastophora rosea } & 0.00 & 0.00 & $0.74 \pm 0.48$ \\
\hline & Apr. 2000 & & \\
\hline Enteromorpha linza & $0.25 \pm 0.30$ & $2.98 \pm 3.60$ & 0.00 \\
\hline Halimeda opuntia & 0.00 & $0.09 \pm 0.24$ & $1.50 \pm 1.41$ \\
\hline Padina australis & 0.00 & $4.14 \pm 3.45$ & $0.00 \pm 0.01$ \\
\hline Sargassum berberifolium & 0.00 & $24.11 \pm 7.06$ & $0.69 \pm 1.20$ \\
\hline Sargassum polycystum & 0.00 & $8.05 \pm 4.24$ & $0.47 \pm 0.93$ \\
\hline Sargassum siliguosum & 0.00 & $7.56 \pm 3.27$ & $0.03 \pm 0.09$ \\
\hline Portieria hornemannii & 0.00 & $0.24 \pm 0.46$ & $1.08 \pm 1.05$ \\
\hline \multirow[t]{2}{*}{ Mastophora rosea } & 0.00 & $0.00 \pm 0.00$ & $0.65 \pm 0.52$ \\
\hline & Aug. 2000 & & \\
\hline Enteromorpha linza & $0.15 \pm 0.21$ & $8.64 \pm 4.16$ & 0.00 \\
\hline Gracilaria coronopifolia & 0.00 & $4.58 \pm 2.34$ & 0.00 \\
\hline Hypnea charoides & 0.00 & $6.79 \pm 3.78$ & 0.00 \\
\hline Portieria hornemannii & 0.00 & $0.04 \pm 0.07$ & $0.95 \pm 1.15$ \\
\hline Mastophora rosea & 0.00 & $0.32 \pm 0.45$ & $0.76 \pm 0.60$ \\
\hline Laurencia papillosa & 0.00 & $4.67 \pm 4.34$ & 0.00 \\
\hline
\end{tabular}

appearance of several algae in October and August, such as Halimeda macroloba, Hydroclathrus clathratus, Hypnea cervicornis, Hypnea charoides and Vidalia obtusiloba. In the upper reef flat, the occurrence of Enteromorpha linza might be also considered as the result of eutrophication. However, the impacts of air-exposure, high temperature and high irradiance can be more profound than the effects of nutrient enrichment for the growth of Enteromorpha linza in the upper reef flat.

The reef slope zone, a subtidal reef flat bordered by a sloping coralline rocky stretch that has a strong undertow is characterized by Halimeda spp. and Mastrophora rosea in all seasons and Portieria hornemannii in April and August 2000. Grazing, irradiance and wave action are considered the factors governing the structure of benthic macroalgal assemblages on the reef slope (Lobban and Harrison 1997).

In conclusion, this study provides evidence that Nanwan coral reefs in southern Taiwan are now characterized by abundant macroalgae, and MDS analyses show the assemblage to be structured primarily by zone and secondarily by season. Although macroalgae are important as primary producers in coral reef systems, overgrowing macroalgae can compete for both space and light with corals and cause adverse effects on the growth of corals in Nanwan Bay. To provide the proper management of benthic communites on southern Taiwan coral reefs, there is need to ascertain the relative importance of abiotic and biotic (such as herbivores) factors affecting the growth of macroalgae.

\section{Acknowledgements}

We are indebted to Prof. Lee-Shing Fang, the President of the National Museum of Marine Biology and Aquarium in Ping-Tung, Taiwan and Prof. Kwang-Tsao Shao, the research fellow in the Institute of Zoology, Academia Sinica, Taipei, Taiwan for their assistance in this study. Our thanks go also to Dr. Shing-Juh Lin in the Department of Life Science, National Chung-Hsing University, Taichung, Taiwan for assisting in non-parametric analysis. We also thank the Kenting National Park of Taiwan of the Republic of China for assistance in field studies. This study was supported by the grants from the National Science Council, Executive Yuan, Taiwan for the collaborative Kenting coral reef conservation study (grants no. 89-2621-Z-110-1 and no. 902621-Z-110-1) and also the Council of Agriculture, Executive Yuan, Taiwan (grants nos. 90AS-1.4.5-FA-F1(22), 91AS-2.5.1F1(13) and 91AS-2.1.4-FC-R2). Comments by Prof. Anthony 
Table 7 Biomass $\left(\mathrm{g} \mathrm{WW} / \mathrm{m}^{2}\right)$ of macroalgal species from SIMPER test.

\begin{tabular}{|c|c|c|c|}
\hline & Upper reef flat & Lower reef flat & Reef slope \\
\hline & Oct. 1999 & & \\
\hline Enteromorpha linza & $2.78 \pm 4.82$ & $126.87 \pm 163.68$ & 0.00 \\
\hline Halimeda macroloba & 0.00 & $2.56 \pm 3.09$ & $3.05 \pm 2.42$ \\
\hline Halimeda opuntia & 0.00 & $0.29 \pm 0.70$ & $2.06 \pm 1.05$ \\
\hline Gracilaria coronopifolia & 0.00 & $49.58 \pm 25.73$ & 0.00 \\
\hline Mastophora rosea & 0.00 & $0.24 \pm 0.48$ & $2.00 \pm 1.79$ \\
\hline Laurencia intermedia & 0.00 & $95.28 \pm 66.10$ & $0.10 \pm 0.29$ \\
\hline Laurencia papillosa & 0.00 & $13.53 \pm 8.42$ & $0.01 \pm 0.02$ \\
\hline \multirow[t]{2}{*}{ Unkown red alga } & $2.29 \pm 2.19$ & $3.07 \pm 4.64$ & 0.00 \\
\hline & Jan. 2000 & & \\
\hline Enteromorpha linza & $9.24 \pm 16.00$ & $54.62 \pm 49.53$ & 0.00 \\
\hline Halimeda macroloba & 0.00 & $25.30 \pm 23.30$ & $15.14 \pm 14.76$ \\
\hline Sargassum berberifolium & 0.00 & $102.26 \pm 115.84$ & 0.00 \\
\hline Sargassum polycystum & 0.00 & $411.98 \pm 405.19$ & $1.34 \pm 2.14$ \\
\hline Gracilaria coronopifolia & $0.18 \pm 0.31$ & $41.56 \pm 29.12$ & $0.25 \pm 0.71$ \\
\hline Hypnea charoides & $4.20 \pm 5.89$ & $62.47 \pm 30.31$ & 0.00 \\
\hline \multirow[t]{2}{*}{ Mastophora rosea } & 0.00 & 0.00 & $13.55 \pm 9.40$ \\
\hline & Apr. 2000 & & \\
\hline Enteromorpha linza & $0.25 \pm 0.30$ & $30.72 \pm 41.52$ & 0.00 \\
\hline Halimeda opuntia & 0.00 & $3.11 \pm 8.91$ & $91.29 \pm 98.62$ \\
\hline Padina australis & 0.00 & $65.13 \pm 61.65$ & $0.12 \pm 0.33$ \\
\hline Sargassum berberifolium & 0.00 & $537.16 \pm 263.60$ & $15.63 \pm 29.05$ \\
\hline Sargassum polycystum & 0.00 & $218.75 \pm 139.33$ & $9.09 \pm 17.89$ \\
\hline Sargassum siliguosum & 0.00 & $302.07 \pm 190.39$ & $0.65 \pm 1.85$ \\
\hline Portieria hornemannii & 0.00 & $11.48 \pm 32.82$ & $63.09 \pm 91.80$ \\
\hline \multirow[t]{2}{*}{ Mastophora rosea } & 0.00 & 0.00 & $30.53 \pm 30.33$ \\
\hline & Aug. 2000 & & \\
\hline Enteromorpha linza & $0.15 \pm 0.21$ & $41.99 \pm 29.90$ & 0.00 \\
\hline Gracilaria coronopifolia & 0.00 & $36.33 \pm 20.02$ & 0.00 \\
\hline Hypnea charoides & 0.00 & $167.66 \pm 109.03$ & $3.75 \pm 10.61$ \\
\hline Portieria hornemannii & 0.00 & $0.36 \pm 0.95$ & $50.93 \pm 94.50$ \\
\hline Mastophora rosea & 0.00 & $3.31 \pm 5.27$ & $41.43 \pm 51.80$ \\
\hline Laurencia papillosa & 0.00 & $12.41 \pm 10.72$ & $0.39 \pm 1.11$ \\
\hline
\end{tabular}

Data are presented as means \pm SE $(n=6)$.

R.O. Chapman in the Biology Department, Dalhousie University, Halifax, NS, Canada and Dr. Bruce G. Hatcher were particularly helpful for revising the manuscript.

\section{References}

Andres, N.G. and J.D. Witman. 1995. Trends in community structure on a Jamaican reef. Mar. Ecol. Prog. Ser. 118: 305-310.

Andrew, N.L. and R.M. Viejo. 1998. Effects of wave exposure and intraspecific density on the growth and survivorship of Sargassum muticum (Sargassaceae: Phaeophyta). Eur. J. Phycol. 33 : 251-258.

Ang, P.O. 1986. Analysis of the vegetation structure of a Sargassum community in the Philippines. Mar. Ecol. Prog. Ser. 28: 9-19.

Banner, A.H. 1974. Kaneohe Bay, Hawaii: urban pollution and a coral reef ecosystem. Proc. 2nd Int. Coral Reef Symp. 2: 685-702.

Bell, P.R.F. 1992. Eutrophication and coral reefs: some examples in the Great Barrier Reef lagoon. Water Res. 26: 553-568.

Bray, J.R. and J.T. Curtis. 1957. An ordination of the upland forest communities of Southern Wisconsin. Ecol. Monogr. 27: 325-349.
Chiang, Y.M. and W.L. Wang. 1988. Ecological studies on marine macroalgae in the Kenting National Park. Conservation Research Report No. 52 of the Kenting National Park, Construction \& Planning Administration of Ministry of Interior, Administration Yuan, Taiwan, Republic of China. pp. 89.

Clarke, K.R. 1993. Non-parametric multivariate analysis of changes in community structure. Aust. J. Ecol. 18: 117-143.

Clarke, K.R. and R.M. Warwick. 1994. Changes in marine communities: an approach to statistical analysis and interpretation. Natural Environment Research Council, Plymouth, UK. pp. 144.

Cuet, P., O. Naim, G. Faure and J.Y. Conan. 1988. Nutrient-rich groundwater impact on benthic communities of La Saline fringing reef (Reunion Island, Indian Ocean): preliminary results. Proc. 6th Int. Coral Reef Symp. 2: 207-212.

Dai, C.F. 1997. Assessment of the present health of coral reefs in Taiwan. In: (R.G. Grigg and C. Birkeland, eds) Status of coral reefs in the Pacific. Sea Grant Program, University of Hawaii. pp. 123-131.

Day, R.W. and G.P. Quinn. 1989. Comparisons of treatments after an analysis of variance in ecology. Ecol. Monogr. 59: 433-463.

DeWreede, R.E. 1976. The phenology of three species of Sargassum (Sargassaceae, Phaeophyta) in Hawaii. Phycologia 15: $175-183$.

Done, T.J. 1992. Phase shifts in coral communities and their ecological significance. Hydrobiologia 247: 121-132.

Hughes, T.P. 1994. Catastrophes, phase-shifts and large scale 
degradation of a Caribbean coral reef. Science 265: 1547-1551.

Hurtado, A.Q. and A.R. Ragaza. 1999. Sargassum studies on Currimao, llocis Norte, Northern Philippines. I. Seasonal variations in the biomass of Sargassum carpophyllum Agardh J., Sargassum llicifolium (Turner) Agardh C., and Sargassum siliquosum Agardh J. (Phaeophyta, Sargassaceae). Bot. Mar. 42: 321-325.

Hwang, R.L., C.C. Tsai and T.M Lee. 2004. Assessment of temperature and nutrient limitation on seasonal dynamics among species of Sargassum from a coral reef in southern Taiwan. J. Phycol. 40: 463-473.

Jephson, N.A. and R.W.G. Gray. 1977. Aspects of the ecology of Sargassum muticum (Yendo) Fenshot in the Solent region of the British Isles. I. The growth cycle and epiphytes. In: (B.F. Keegan, P.O. Ceidgh and P.J.S. Boaden, eds) Biology of benthic organisms. Pergamon Press, Oxford. pp. 367-375.

Kruskal, J.B. and M. Wish. 1978. Multidimensional scaling. Sage Publications, Beverly Hills, California. pp 93.

Lambshead, P.J.D., H.M. Platt and K.M. Shaw. 1983. The detection of differences among assemblages of marine benthic species based on an assessment of dominance and diversity. J. Nat. Hist. 17: 859-874.

Lapointe, B.E. 1997. Nutrient thresholds for bottom-up control of macroalgal blooms on coral reefs in Jamaica and southeast Florida. Limnol. Oceanogr. 42: 1119-1131.

Lapointe, B.E. and J.O'Connell. 1989. Nutrient-enhanced growth of Cladophora prolifera in Harrington Sound, Bermuda: eutrophication of a confined, phosphorus-limited ecosystem. Estuar. Coast. Shelf Sci. 28: 347-60.

Littler, M.M. and D.S. Littler. 1980. The evolution of thallus form and survival strategies in benthic marine macroalgae: field and laboratory tests of a functional form model. Am. Nat. 116: $25-44$

Littler, M.M., D.S. Littler and E.E. Lapointe. 1992. Modification of tropical reef community structure due to cultural eutrophication: the southwest coast of Martinique. Proc. 7th Int. Coral Reef Symp. 1: 335-343.

Lobban, C.S. and P.J. Harrison. 1997. Seaweed ecology and physiology. Cambridge University Press, New York. pp. 366.
Lüning, K. 1993. Environmental and internal control of season growth in seaweeds. Hydrobiologia 260/261: 1-14.

McCook, L.J. 1999. Macroalgae, nutrients and phase shifts on coral reefs: scientific issues and management consequences for the Great Barrier Reef. Coral Reefs 18: 357-367.

McCook, L.J., J. Jompa and G. Diaz-Pulido. 2001. Competition between corals and algae on coral reefs: a review of evidence and mechanisms. Coral Reefs 19: 400-417.

Murphy, J. and J.P. Riley. 1962. A modified single solution method for the determination of phosphate in natural waters. Anal. Chim. Acta 27: 31-36.

Naim, O. 1993. Seasonal responses of a fringing reef community to eutrophication (Reunion Island, Western Indian Ocean). Mar. Ecol. Prog. Ser. 99: 137-151.

Parsons, T.R., Y. Maita and C.M. Lalli. 1984. A manual of chem$i c a l$ and biological methods for seawater analysis, Pergamon Press, New York. pp. 173.

Pielou, E.C. 1975. Ecological diversity. John Wiley \& Sons, New York. pp. 165.

Rodriguez-Prieto, C. and L. Polo. 1996. Effects of sewage pollution in the structure and dynamics of the community of Cystoseira mediterranea (Fucales, Phaeophyceae). Sci. Mar. 60: 253-263.

Schaffelke, B. and D.W. Klumpp. 1997. Biomass and productivity of tropical macroalgae on three nearshore fringing reefs in the central Great Barrier Reef, Australia. Bot. Mar. 40: 373-383.

Shannon, C.E. and W. Weaver. 1949. The mathematical theory of communication. University of Illinois Press, Urbana, IL. pp.177.

Smith, V.R., W.J. Kimer, E.K. Laws, R.E. Brock and T.W. Walsh. 1981. Kaneohe Bay sewage diversion experiment: perspectives on ecosystem responses to nutrient perturbation. Pac. Sci. 35: 379-402.

Strickland, J.D.H. and T.R. Parsons. 1972. A practical handbook of sea water analysis. Fish. Res. Board Can. Bull. 17: 1-310.

Wang, W.L. and Y.M. Chiang. 1994. Potential economic seaweeds of Hengchun Peninsula, Taiwan. Econ. Bot. 48: 182-189.

Received 13 January, 2003; accepted 28 September, 2004 